wendigkeiten abdeckt. Zugleich haben die reisebezogenen Ansprüche im Laufe des 20. Jahrhunderts an Bedeutung eingebüßt. War es im 19. Jahrhundert vielen Reichstagsabgeordneten ohne privates Vermögen nicht möglich, an Reichstagssitzungen teilzunehmen, da sie keine Diäten erhielten und während mehrerer Sessionen auch keine Freifahrtberechtigung besaßen, ist deren Wichtigkeit in dem Maße gesunken, wie die Entschädigung erhöht wurde und den Lebensunterhalt zu sichern vermochte. Auch an dieser im Vergleich zu anderen abgeordnetenrechtlichen Leistungen gestiegenen Bedeutung der Entschädigung zeigt sich die fortschreitende Professionalisierung der Abgeordnetentätigkeit.

Das Abgeordnetenrecht des Bundes und der Länder wurde seit der Gründung der Bundesrepublik viele Male modifiziert. Weitere Änderungen werden sicherlich folgen. Die Empfehlungen der Unabhängigen Kommission zu Fragen des Abgeordnetenrechts vom 19. März 2013 haben - wie gezeigt - zu deutlichen Änderungen geführt. Aber auch sie werden wohl nicht die letzten sein. Die häufig allzu erregten Debatten um die Höhe und die Ausgestaltung der finanziellen und geldwerten Leistungen an Abgeordnete gibt es schon seit der Weimarer Zeit. Sie werden mit Sicherheit auch in Zukunft weitergehen. Die Parlamente können sie selbstbewusst führen: Ein angemessenes Einkommen und eine entsprechende Amtsausstattung der Abgeordneten sind nicht Ausdruck irgendeiner Selbstbedienungsmentalität, sondern unverzichtbare Voraussetzungen wirklicher Demokratie und erfolgreicher parlamentarischer Repräsentation. Sie ermöglichen die Teilhabe aller am Staatsgeschehen und sichern die für moderne demokratische Gesellschaften unumgängliche Professionalität des Mandats.

\title{
Unerfreuliche Einblicke in Tiefenschichten deutscher Parlaments- wahrnehmung: Die Kritik an den Vorschlägen der Unabhängigen Kommission zu Fragen des Abgeordnetenrechts
}

\author{
Werner J. Patzelt
}

\section{Zur Leistung der Kommission}

Die im November 2011 vom Ältestenrat des Bundestages eingesetzte „Unabhängige Kommission zu Fragen des Abgeordnetenrechts“ war fleißig. Zwischen Dezember 2011 und März 2013, als sie ihren Bericht dem Bundestagspräsidenten übergab ${ }^{1}$, tagte sie nicht weniger als 17-mal im vertraulichen Kreis ihrer elf Mitglieder, hörte 13 Experten und arbeitete sich, gestützt auf ein vierköpfiges Sekretariat, durch das einschlägige, auch innerstaatlich und international vergleichende rechts- sowie politikwissenschaftliche Schrifttum, durch

1 „Bericht und Empfehlungen der Unabhängigen Kommission zu Fragen des Abgeordnetenrechts“, veröffentlicht als BT-Drs. 17/12500 vom 19. März 2013, ebenso in: Suzanne S. Schüttemeyer / Edzard Schmidt-Jortzig (Hrsg.), Der Wert der parlamentarischen Repräsentation. Entwicklungslinien und Perspektiven der Abgeordnetenentschädigung, Baden-Baden 2013, S. 17 - 102. Eine Einschätzung der Arbeit und Leistung der Expertenkommission aus der Sicht ihres Vorsitzenden findet sich unter anderem in einem Interview mit Edzard Schmidt-Jortzig, in: Das Parlament vom 22. April 2013 sowie in seinem Beitrag in diesem Heft der ZParl. 
die relevante verfassungsgerichtliche Rechtsprechung, obendrein durch die Berichte früherer Kommissionen. ${ }^{2}$ Ihren Bericht legte sie auch noch binnen der vorgesehenen Frist vor, nämlich am 18. März 2013, und löste so obendrein eine - zumal in den Reihen der Union geführte - Debatte darüber aus, ob wohl noch der „alte“ oder erst der im Herbst neu zu wählende Bundestag sich mit ihren Empfehlungen befassen solle. ${ }^{3}$

Außerdem arbeitete die Kommission ehrgeizig und umsichtig. Am Anfang ihres Berichts - nach den zusammenfassenden Leitsätzen sowie Angaben über die eigene Zusammensetzung, Aufgabenstellung und Arbeitsweise - steht eine vortreffliche Analyse der Funktionslogik unseres Parlamentarismus und der Anforderungen an Abgeordnete („Leitbild: Parlament und Abgeordnete unter dem Grundgesetz“, „Bedeutung des Bundestages und des Abgeordnetenamtes im Verfassungsgefüge" $)^{4}$, die sich auch gut als politikwissenschaftlicher Lehrbuchtext eignen könnte und von der ...

„sich eben alle Ansätze und Empfehlungen ableiten. Nur so kann jede Argumentation überhaupt nur schlüssig sein. Und alle Kritik an Einzelpunkten muss sich dann fragen lassen, wo sie denn in der Prämisse und/oder dem Deduktionsgang nun einen Fehler sieht. Das ,Leitbild' hat dabei selbstverständlich jeder Überprüfung standzuhalten, ob es nicht womöglich von den Interpreten selbst eingefärbt oder stilisiert wird, sondern sich immer belastbar aus den normativ-funktionalen Vorgaben herleitet" ${ }^{\text {" }}$.

Im Anschluss an dieses „Leitbild“ findet sich eine Erörterung der bestehenden verfassungsrechtlichen Vorgaben für eine angemessene Abgeordnetenbesoldung, die das Nachdenken über die zu beachtende Rechtslage aus den seit 1975, dem Jahr des weichenstellenden „Diätenurteils“ des Bundesverfassungsgerichts ${ }^{6}$, eingefahrenen Gleisen befreit. ${ }^{7}$ Danach wird

$2 \mathrm{Zu}$ diesen siehe Harro Semmler, Externer Sachverstand bei Entscheidungen in eigener Sache. Unabhängige Kommissionen und Gremien zur Rechtsstellung der Abgeordneten des Deutschen Bundestages, in: Suzanne S. Schüttemeyer / Edzard Schmidt-Jortzig (Hrsg.), a.a.O. (Fn. 1), S. 147 -171 .

3 Bundestagspräsident Norbert Lammert befürwortete eine Neuregelung noch vor der Wahl, um nicht den nächsten Bundestag seine Arbeit gleich mit einer Erhöhung der Diäten beginnen zu lassen, während die eher taktisch denkenden Parteipolitiker derlei nicht zum Wahlkampfthema machen und deshalb dem neuen Bundestag überlassen wollten. Siehe hierzu etwa Robert Rossmann, Unionsfraktion gegen Lammert, in: SZ vom 3. April 2013, S. 6; Karl Doemens, Union will Diäten vorerst nicht verändern, in: FR vom 3. April 2013, S. 4; Margarete van Ackeren / Stephanie E. Stallmann / Herbert Rossler-Kreuzer, Null-Diät ist noch zu viel, in: Focus vom 8. April 2013, S. $36 \mathrm{f}$. Der Bundestagspräsident meinte zu alledem in einem Interview mit der Leipziger Volkszeitung vom 1. April 2013: „Jedenfalls ist der Mut der Abgeordneten, zu allgemeinen Dingen gelegentlich unpopuläre Beschlüsse zu fassen ... erkennbar ausgeprägter, als die Bereitschaft, in eigenen Angelegenheiten unpopuläre Entscheidungen zu treffen." Ganz kontrafaktisch sieht das eine Mehrheit der Deutschen offenbar anders und wirft den Parlamentariern gemeinplatzartig „Selbstbedienungsmentalität" vor; dazu unten mehr.

4 Bericht und Empfehlungen, in: Suzanne S. Schüttemeyer / Edzard Schmidt-Jortzig (Hrsg.), a.a.O. (Fn. 1), S. $24-30$, S. $36-44$.

5 Edzard Schmidt-Jortzig, Die „Unabhängige Kommission zu Fragen des Abgeordnetenrechts“: orientiert an realistischen Normen und parlamentarischer Praxis, in: Suzanne S. Schüttemeyer / ders. (Hrsg.), a.a.O. (Fn. 1), S. 9 - 16, S. 11. Der nachfolgende Blick auf die Kritik am Kommissionsbericht wird zeigen, dass Medien und Öffentlichkeit überhaupt nicht auf diese beabsichtigte Weise mit dem „Leitbild“ umgingen, sondern sich dieses bisweilen sogar ironisiert und karikiert fand.

6 BVerfGE 40, S. $296 \mathrm{ff}$.

7 Vgl. Bericht und Empfehlungen, in: Suzanne S. Schüttemeyer / Edzard Schmidt-Jortzig (Hrsg.), a.a.O. (Fn. 1), S. 33 - 36, S. 52 f. 
der bestehende Regelungsbestand zur Abgeordnetenentschädigung, zur Amtsausstattung, zum Übergangsgeld, zur Altersentschädigung und zur Absicherung außerparlamentarischer Berufstätigkeit geschildert. ${ }^{8}$ Und abgeleitet aus dem - anhand jenes „Leitbilds“ - systematisch erörterten Soll, hineingestellt in einen durch juristische Hermeneutik neu erschlossenen Möglichkeitsraum sowie bezogen auf das - auch seinerseits vom „Leitbild“ her positiv geschilderte - Ist gelangt die Kommission sodann zu einer Reihe von sorgfältig nach Für und Wider begründeten Empfehlungen.

Einhellig riet die Kommission, die Grundbezüge von Abgeordneten - wie schon jahrelang vorgesehen ${ }^{9}$ - am Einkommen von Richtern an obersten Gerichtshöfen des Bundes auszurichten ${ }^{10}$ sowie jährlich an die durch den „Nominallohnindex“ gemessene Entwicklung der Bruttomonatsverdienste der abhängig Beschäftigten im Bundesgebiet anzupassen. Über eine Beibehaltung dieser - fortan im Abgeordnetengesetz zu verankernden - Indexierung solle der Bundestag, darin weiterhin dem Verfassungsgerichtsurteil von 1975 folgend $^{11}$, zu Beginn jeder Wahlperiode mit Wirkung für deren Rest entscheiden. Einhellig empfahl man auch, Funktionsvergütungen für Ausschussvorsitzende und Inhaber von Fraktionsämtern durch Aufnahme ins Abgeordnetengesetz aus ihrer rechtlichen Grauzone zu holen, und fraktionsinterne Zulagen, ihrerseits im Zuständigkeitsbereich der Fraktionsautonomie, einer strengeren Berichtspflicht zu unterstellen. Keine einhellige Empfehlung gab es zur künftigen Ausgestaltung des Altersversorgungssystems für Abgeordnete. Hier wurde, auf gemeinsam unterstützten Kriterien basierend, dem Bundestag eine Alternative zur Auswahl vorgeschlagen: (modifizierte) Beibehaltung des derzeitigen Systems - oder Einführung eines „Bausteinmodells“, bestehend aus Weiternutzung bisheriger Versorgungsansprüche, zusätzlicher Einzahlung in die gesetzliche oder eine andere Rentenkasse sowie sonstiger Eigenvorsorge. ${ }^{12}$ Nicht einhellig waren auch die - ohnehin nicht im Auftrag an die Kommission verlangten - Empfehlungen zur Kostenpauschale. Der Mehrheit schien es richtig zu sein, die bisherige Praxis einer reinen Pauschale beizubehalten, während die Minderheit vorschlug, typisierbare mandatsbedingte Kosten (wie die Mieten für eine Unterkunft in Berlin oder für Wahlkreisbüros) bis zu einer angemessenen Obergrenze gegen Nachweis zu erstatten und nur die - dann klar reduzierten - restlichen Ausgaben pauschal abzugleichen.

Mit ihren einhelligen Vorschlägen zeigte die Kommission den Bundestagsabgeordneten einen Weg auf, künftig den Eindruck maßstabslosen Erwerbstriebs in nie endenden Selbst-

8 Vgl. ebenda, S. $44-51$.

9 Nämlich im Entwurf eines Gesetzes zur Neuregelung der Rechtsstellung der Abgeordneten vom 28. November 1995, BT-Drs. 13/3121, S. 8.

10 Etwa am Bundesgerichtshof oder am Bundesverwaltungsgericht; Besoldungsgruppe R 6; weitere Kriterien der Gehaltsbemessung: verheiratet, Familienzuschlag, Zulage für oberste Bundesbehörden.

11 Siehe hierzu insbesondere BVerfGE 40, S. 296 (S. 327): „In einer parlamentarischen Demokratie lässt es sich nicht vermeiden, dass das Parlament in eigener Sache entscheidet, wenn es um die Festsetzung der Höhe und um die nähere Ausgestaltung der mit dem Abgeordnetenstatus verbundenen finanziellen Regelungen geht. Gerade in einem solchen Fall verlangt aber das demokratische und rechtsstaatliche Prinzip (Art. 20 GG), dass der gesamte Willensbildungsprozess für den Bürger durchschaubar ist und das Ergebnis vor den Augen der Öffentlichkeit beschlossen wird. Denn dies ist die einzige wirksame Kontrolle."Wie längere Zeiten der Nichtanpassung der Diäten an die allgemeine Einkommensentwicklung zeigen (nämlich 1977 bis 1983, 1992 bis 1995, 2003 bis 2007), funktionierte diese Kontrolle recht effektiv.

12 Ein Mitglied der Kommission plädierte für eine reine Eigenvorsorge. 
bereicherungsrunden zu vermeiden. Ihren Argumenten dogmatischer oder pragmatischer Art, auch ihrem auf sorgfältigem Abwägen anderer Problemlösungsmöglichkeiten gegründeten Vorschlag, ist vom Verfasser nichts Besseres hinzuzufügen. ${ }^{13}$ Die Empfehlung, einen gewissen Automatismus bei der Gehaltsanpassung von Abgeordneten einzuführen, war angesichts der aufgrund vergangener Erfahrungen zu erwartenden massiven öffentlichen Ablehnung - im Übrigen von ebenso lobenswertem Mut wie der Vorschlag, im Parlament selbst zu einer rechtlich unumstrittenen aufgaben-, positions- beziehungsweise verantwortungsbezogenen Einkommensdifferenzierung unter den Abgeordneten zu gelangen. ${ }^{14}$ Bei ihren nicht einhelligen Vorschlägen lässt sich der Kommission hingegen teils Festhalten an dem ankreiden, was bislang Mehrheitskonsens ist, teils Abschiebung schon der Vorentscheidungslast an den Bundestag: ersteres bei der Kostenpauschale, letzteres bei der Altersversorgung. Doch gerechterweise ist hinzuzufügen, dass es sich gerade bei der Altersversorgung um eine überaus komplexe Regelungsmaterie handelt, bei der die Provokation parlamentarischen Widerstands gegen einen klaren Kommissionsvorschlag das Risiko nach sich gezogen hätte, auch für die Neuregelung der Abgeordnetenbesoldung - Kern der Kommissionsvorschläge - keine Mehrheit im Bundestag zu finden. Erst recht galt letzteres für einen klaren Neuregelungsvorschlag zum praktischen Kernbereich höchstpersönlicher Amtsführung, nämlich zur Kostenpauschale. Bemüht, überhaupt in der Sache voranzukommen, beschränkte sich die Kommission weise auf das halbwegs Erfolgversprechende. Also bleiben mancherlei Reformwünsche unerfüllt. Die werden sich hinsichtlich ihrer offenen Streitund Abwägungsfragen wohl erst durch Wachsenlassen der anstehenden Probleme einer einhelligen Antwort näherbringen lassen. So ist es in der Politik ohnehin sehr oft, und so war es ebenfalls bei den Neuregelungen zur parlamentsinternen Einkommensdifferenzierung sowie beim nunmehr gefundenen „Algorithmus“ der Gehaltsanpassung.

Mit ihren einhelligen Empfehlungen hatte die Kommission jedenfalls, anders als so manche Vorgängerkommission ${ }^{15}$, auch Erfolg: Am 21. Februar 2014, knapp ein Jahr nach Abgabe ihres Berichts, entsprach ihnen der Bundestag durch Beschluss eines weiteren Gesetzes zur Änderung des Abgeordnetengesetzes. Künftig dient als - in zwei (2014 und 2015 erfolgenden) Diätenerhöhungen zu erreichende - Ausgangsgröße des Abgeordnetengehalts

13 Siehe insbesondere Bericht und Empfehlungen, in: Suzanne S. Schüttemeyer / Edzard SchmidtJortzig (Hrsg.), a.a.O. (Fn. 1), S. 52 - 66. Zu den erwogenen Alternativen vgl. Suzanne S. Schüttemeyer / Johannes Haas, Abgeordnetenbezüge im internationalen Vergleich, in: ebenda, S. $173-196$.

14 Allerdings wäre vor dem Hintergrund genau dieser leistungsbezogenen parlamentsinternen Differenzierung eine ausdrückliche Argumentation dazu wünschenswert gewesen, warum denn das sozusagen - „Einstiegsgehalt“ eines Abgeordneten auf jener Höhe liegen müsse, die bei Richtern an den obersten Bundesgerichten oder bei Bürgermeistern mittelgroßer Städte mit 50.000 bis 100.000 Einwohnern meist das Endgehalt darstellt - eine Kritik, die sich zumal in Blogs findet, etwa dem folgenden von „Mami“ in: „Abgeordnetenwatch“ vom 21. Februar 2014: „Einfache Abgeordnete mit befähigten Juristen in der Gehaltsstufe gleichzusetzen, finde ich fatal. Viele Juristen haben jahrelang ihr Fach studiert und dann bei den entsprechenden Ministerien ihre zweite juristische Verwaltungsprüfung, manchmal sogar mit einem Prädikat, abgelegt. Wer schon kann das von manchen (vielen?) Bundestags-Abgeordneten, die ja auch für unser Recht eintreten sollen, erwarten?" (Schreibfehler im Original).

15 Etwa der Kissel-Kommission (benannt nach ihrem Vorsitzenden Otto Rudolf Kissel, damals Präsident des Bundesarbeitsgerichts), die im Juni 1992 berufen wurde und ein Jahr später einen umfangreichen Bericht vorlegte. 
die Besoldung der Richter an einem obersten Gerichtshof des Bundes (mit amtsbezogener Zulage, doch ohne Familienzuschlag); ab 2016 wird das Abgeordneteneinkommen nicht mehr „freihändig“, sondern gemäß der vom Statistischen Bundesamt ermittelten durchschnittlichen Veränderung der Nominallöhne angepasst; fortan gibt es Zulagen auch für die Vorsitzenden der ständigen Ausschüsse, Unterausschüsse und Enquete-Kommissionen, nämlich bis zu 15 Prozent eines Monatsgehalts; und ansonsten bleibt es im Wesentlichen beim bisherigen Zustand, außer dass kleinere Abstriche bei der Altersversorgung gemacht werden, die künftig allerdings von einem höheren Grundeinkommen ausgeht. ${ }^{16}$

\section{Die Kritik an den Kommissionsempfehlungen als Erkenntnisquelle}

Viel weniger erfolgreich war die Diätenkommission darin, einer breiteren Öffentlichkeit auch den Verständnisrahmen ihrer Empfehlungen zu vermitteln, nämlich ihr „Leitbild“ von Parlament und Abgeordneten. Weil aber die Kommissionsempfehlungen nun einmal auf dieses „Leitbild“ ausgerichtet sind, stießen sie bei einem beachtlichen Teil der Öffentlichkeit nicht nur auf achselzuckende Ablehnung, sondern lösten gar eine Art Wutschnauben aus. ${ }^{17}$ In ihm äußert sich jene - aus höchst traditionellen Quellen gespeiste ${ }^{18}$ - Kritik an den Leitgedanken und Empfehlungen der Expertenkommission, mit deren recht vorhersehbaren Inhalten sich von vornherein ausdrücklich, ja offensiv auseinanderzusetzen die Kommission in ihrem Bericht unterlassen hat. Zwar wurden viele dieser Kritikpunkte implizit - und ganz offenbar auch absichtlich - im systematisch entwickelten „Leitbild“ aufgegriffen, wo sie durch die ganze Anlage der dort entwickelten Argumente für unerheblich erklärt werden. Doch eine Beschlussempfehlung für den Bundestag erstellend, nicht aber einen Beitrag zur politischen Bildung verfassend, unterließ es die Kommission, ihre Empfehlungen Punkt für Punkt jener Kritik gegenüberzustellen, die sich an der Abgeordnetenbesoldung doch regelmäßig entzündet. Was von der Kommission vorgeschlagen wurde, wirkt deshalb zwar im Argumentationshorizont des „Leitbilds“ plausibel, zumal für Parlamentarismusexperten. Es werden aber keinem Kritiker der bisherigen - oder der neu empfohlenen - Regelungen irgendwelche Argumentationslasten auferlegt, die über eine bloße

16 Obendrein wurde, im Grunde nicht mehr als ein symbolischer Akt, auch noch die bereits seit 1994 in $\$ 108$ e des Strafgesetzbuches verbotene Abgeordnetenbestechung in $\$ 44$ a des Abgeordnetengesetzes untersagt. Diese lange schon erhobene Forderung war nicht Gegenstand der Kommission gewesen, wurde aber gleich nach Bekanntwerden des Kommissionsberichts erst recht vorgebracht, besonders lautstark etwa vom Grünen-Abgeordneten Volker Beck, siehe Robert Rossmann, a.a.O. (Fn. 3).

17 Noch der geringste Teil der Kritik bezog sich darauf, dass es der Kommission bei der Altersversorgung und der Kostenpauschale nicht gelungen war, zu einem gemeinsamen Vorschlag zu kommen; siehe etwa Karl Doemens, a.a.O. (Fn. 3) und seinen Kommentar über „Lammerts Diätenreform“ in der gleichen Ausgabe, S. 13: „Die von ihm [Lammert] eingesetzte Expertenkommission hat versagt: Überall dort, wo Veränderungen zulasten der Abgeordneten nötig wären, kam sie zu keinem Ergebnis.“ Weniger scharf, nämlich nur als „halbherzig“, kritisierte Thomas Maron, Wenn schon, dann richtig, in: Stuttgarter Zeitung vom 3. April 2013, S. 3, die Ratschläge der Expertenkommission.

18 Siehe hierzu unten den Abschnitt 5 und die dortigen Literaturverweise sowie Philipp Austermann, Die Geschichte der Abgeordnetenentschädigung, in: Suzanne S. Schüttemeyer / Edzard SchmidtJortzig (Hrsg.), a.a.O. (Fn. 1), S. $103-145$. 
Wiederholung bisheriger Argumentationsfiguren hinausgingen. Das ist zwar der Aufgabe der Kommission und der Textsorte ihres Berichts geschuldet und gewiss nicht einem Mangel an Argumenten. Doch wer, aus welchen Gründen auch immer, sich mit dem „Leitbild“ als Kern der Kommissionsposition nicht ernsthaft auseinandersetzen wollte, der bekam so einen recht einfach zu nutzenden Hebel in die Hand: Er konnte argumentieren, die Kommission sei ohnehin unausgewogen zusammengesetzt, sozusagen „nur aus Abgeordnetenfreunden“, vertrete deshalb parteiisch eine zugunsten der Parlamentarier tendenziöse Position, und deshalb brauche man sich auf ihr „Leitbild“ gar nicht erst einzulassen. ${ }^{19}$

Es ist nachrangig, ob die Kommission, die konkret erwartbaren Kritikpunkte durchaus antizipierend, diese einfach - in des Wortes dialektischer Bedeutung - „im Leitbild aufheben“ wollte, oder ob sie, die Textsorte eines Beschlussvorschlags für den Bundestag achtend, die Gemeinplätze einschlägiger Kritik nicht dadurch „adeln“ mochte, dass sie sich auch explizit auf sie einließ. Was hinsichtlich der Außenwirkung des Kommissionsberichts zählt, ist nämlich das politisch-kulturelle Ergebnis: Außerhalb von Union und SPD stieß auf teils reflexartige, teils opportunistische Kritik, was von der Kommission doch mit sehr differenzierten und dadurch zur sorgfältigen Auseinandersetzung einladenden Begründungen vorgeschlagen wurde. Eben dieses Phänomen ist nun besonders erhellend: Es lässt Tiefenschichten deutscher Parlamentarismuskritik erkennen, auch bis hin zur Unbelehrbarkeit verfestigte Vorurteile gegen Abgeordnete und Parlamente, ja sogar argumentative Untiefen selbst bei Kundigen und Wohlmeinenden. ${ }^{20}$

Eben das zeigt die nachstehende Untersuchung. Ihre Materialgrundlage ist im Wesentlichen der vom Deutschen Bundestag zusammengestellte Pressespiegel zum Medienecho auf den Bericht der Expertenkommission zwischen dem 19. März, dem Tag nach Übergabe des Kommissionsberichts, und Ende April 2013. Ergänzend wurden repräsentative demoskopische Befunde sowie exemplarische Aussagen in Blogs und Diskussionsforen über Abgeordnete herangezogen. ${ }^{21}$ Ziel der Analyse ist dabei nicht die inhaltliche Auseinandersetzung mit der dokumentierten Kritik, wenngleich des Verfassers Position zu alledem stets deutlich wird. Vielmehr geht es um die Interpretation der vorgebrachten Einwände im größeren Rah-

19 Siehe hierzu unten den Abschnitt 4.3.

20 Siehe zu diesem politisch-kulturellen Verständnishorizont Werner J. Patzelt, Ein latenter Verfassungskonflikt? Die Deutschen und ihr parlamentarisches Regierungssystem, in: PVS, 39. Jg. (1998), H. 4, S. 725 - 757; ders., Warum verachten die Deutschen ihr Parlament und lieben ihr Verfassungsgericht? Ergebnisse einer vergleichenden demoskopischen Studie, in: ZParl, 36. Jg. (2005), H. 3, S. $517-538$.

21 Wenig überraschend sind die Diskussionen in Blogs und Foren recht einseitig und fallen fast immer zu Ungunsten der Parlamentarier aus. Beiträge, die eine Erhöhung der Abgeordnetenbezüge unterstützen, sind deutlich in der Minderheit. Auf „Abgeordnetenwatch“, http://spiegel. abgeordnetenwatch.de/diaetenerhoehung-1105-557----all.html\#comments (Abruf am 19. März 2014), gab es beispielsweise über 200 Kommentare zum Abstimmungsverhalten der Abgeordneten bei der Diätenerhöhung dieses Jahres, darunter aber keinen einzigen, der die Erhöhung befürwortete. Zeigte sich in solchen Blogs und Foren ein grundsätzlich anderes Bild als in Leserbriefen und demoskopischen Befunden, könnte man diese Form hochgradig selektiver Öffentlichkeit als für einen Blick in die Tiefenschichten deutschen Parlamentsdenkens unbeachtlich abtun. Da es so eben nicht ist, wäre eine systematische Inhaltsanalyse einer repräsentativen Stichprobe entsprechender Foren- und Blogbeiträge wünschenswert, lag aber außerhalb der Möglichkeiten dieses Beitrags. In ihm runden die entsprechenden Zitate plastisch ab, was sich in den anderen Quellen ebenso zeigt. 
men bundesdeutscher Parlaments- und Abgeordnetenkritik. Dafür ist es wichtig, sorgfältig auf den - umfangreich dokumentierten - „O-Ton Volk“ zu hören. Er klingt nämlich oft erschreckend, ist aber - wie sein Vergleich mit repräsentativen demoskopischen Befunden zeigt - nur die markante Spitze eines Eisbergs normalerweise unbeachteter, gleichwohl aber delegitimierend-folgenreicher Alltagskommunikation. Derartige Einlassungen, darunter auch Entgleisungen, akademisch überhören zu wollen, etwa weil „niveaulo“ oder „ohne Substanz“, führt deshalb nicht weiter. Vielmehr zeigen jene Zitate zwei wichtige Aufgabenbereiche an: Inhaltlich wird klar, wo weitere Bemühungen um bessere politische Bildung der Bevölkerung ansetzen müssen; und politisch zeigt sich, wie dringlich konkrete Gedanken darüber sind, auf welche Weise wir gerade jene Abgeordneten bekommen könnten, die auch im Urteil der Bevölkerung das verdienen dürften, was sie laut Urteil der Diätenkommission verdienen sollten.

\section{Positive Würdigungen des Kommissionsberichts - und einige Reaktionen auf sie}

Nicht alle Urteile über die Empfehlungen der Expertenkommission waren allerdings negativ. ${ }^{22}$ Der Mannheimer Morgen etwa überschrieb seine entsprechende Meldung vom 20. März 2013 einfach mit „Sinnvoll“ und formulierte: „Die Idee ist nicht neu, aber sie hat Charme. Die Bezahlung der Bundestagsabgeordneten soll sich künftig an der Besoldung von Bundesrichtern orientieren ... [so dass] damit endlich die Debatte um Diätenerhöhungen vom Tisch" wäre. ${ }^{23}$ Kritisiert wird allein, dass sich nicht ebenfalls für die Altersversorgung ein einhelliger Reformvorschlag vorgelegt fand. Selbst der das Werk der Expertenkommission ansonsten heftig kritisierende Karl Doemen meinte in seinem Kommentar zu „Lammerts Diätenreform“ vom 3. April 2013 in der Frankfurter Rundschau:

„Eine Demokratie braucht qualifizierte Volksvertreter, die angemessen bezahlt werden. Prinzipiell ist der Versuch, einen objektiven Maßstab für die Entwicklung der Abgeordnetendiäten zu finden, daher nicht verwerflich."

Freundlicher las sich das in der Rheinischen Post vom 3. April 2013: „Die Reformvorschläge sind daher gut. Weg mit der Pflicht, ständig selbst über die eigenen Diäten entscheiden

$22 \mathrm{Zu}$ den positiven oder immerhin neutralen Urteilen gehören, neben den unten zitierten, vor allem Günter Bannas, Vom Bundestag beschlossene Diäten, in: FAZ vom 4. April 2013, S. 4, sowie Donata Riedel, Der Wert des Parlaments, in: Handelsblatt vom 3. April 2013, S. 14. Gelobt wird dort, dass die Kommission die Abgeordnetenbesoldung künftig an klare - und nach Ansicht der Autorin: auch angemessene - Kriterien binden will; kritisiert wird hingegen, dass die Kommission „kein überzeugendes Maß“ für die „großzügigen Übergangsregelungen und frühen Pensionszahlungen" gefunden habe. Ebenso fehle es an geforderter Transparenz bei den Honoraren für Nebentätigkeiten.

23 Martin Ferber, Sinnvoll, in: Mannheimer Morgen vom 20. März 2013. Auch Bundestagspräsident Lammert betonte in der FAZ vom 2. April 2013, S. 4, eine der wichtigen Aufgaben bestehe „ja gerade darin, nach Regelungen zu suchen, die diesen Verdacht der Selbstbedienung reduzieren können“. Ferner versuchte er mehrfach ins Bewusstsein zu heben: „Bei der Besoldungshöhe empfiehlt die Kommission keine neue Größe, sondern rät uns, endlich umzusetzen, was seit 20 Jahren im Abgeordnetengesetz vorgesehen ist" (zitiert nach Annett Meiritz, Diäten-Debatte: Lammert favorisiert höhere Abgeordnetengehälter, in: Spiegel Online vom 1. April 2013, http://www.spiegel.de/politik/deutschland/abgeordnete-bundestagspraesident-lammert-liebaeugelt-mit-hoeherendiaeten-a-891911.html, Abruf am 4. Juni 2014). 
zu müssen, mehr Flexibilität für mehr Leistung“. Und nachgerade ein Übersoll an Zustimmung formulierte in der „Welt" vom 3. April 2013 Dorothea Siems:

„Die Volksvertreter sind hierzulande keineswegs überbezahlt. Ihren Verdienst an die Besoldung von Bundesrichtern anzupassen ... ist sogar eher zu bescheiden als zu großzügig. Schließlich ist das politische Tagesgeschäft anspruchsvoll und zeitaufwendig. Hinzu kommt das strapaziöse Pendeln zwischen Hauptstadt und Wahlkreis. “24

Grundsätzlich zustimmend, allerdings auf „Ja, aber ..."-Basis, war ferner ein - so auch untertitelter - Beitrag im Berliner Kurier vom 5. April 2013. Dort wurden drei Bedingungen für die Akzeptabilität der von der Expertenkommission vorgeschlagenen Neustrukturierung der Abgeordnetenbezahlung gestellt: Abgeordnetenbestechung wird künftig wie bei Beamten bestraft; die Kostenpauschale wird durch Kostenerstattung auf Nachweisbasis ersetzt; und die Altersversorgung wird reformiert.

Doch sich öffentlich klar positiv zu den Vorschlägen der Expertenkommission zu äußern, konnte auch zum eigenen Nachteil ausschlagen - und zwar nicht nur für Politiker, denen als den „Begünstigten“ so mancher ohnehin kein angemessenes Urteil zutraut. ${ }^{25}$ Insbesondere Peter Hahne musste Federn lassen, nachdem er am 24. März 2013 in der Bild am Sonntag seine Kolumne mit „Abgeordnete sind keine Räuberbande“ überschrieben und dann ausgeführt hatte:

„Auf keinen Beruf wird so gnadenlos öffentlich eingedroschen wie auf den des Politikers. Empörungswellen im Internet und pauschales Abwatschen der Volksvertreter ... sind fast schon ein beliebter Volkssport. Merken wir gar nicht, dass wir damit das Kostbarste zerstören, das ,Unsere Mütter, unsere Väter' nach der Katastrophe des Zweiten Weltkriegs aufgebaut haben: Die beste Demokratie der Welt, repräsentiert durch frei gewählte Abgeordnete. $^{26} \ldots$

Kaum gemeldet [nämlich: die Empfehlungen der Kommission] schon der Aufschrei. Als seien Parlamentarier durchweg Absahner und Abzocker, faul und korrupt, geht ein Tsunami der Entrüstung durchs Land. Selbstbedienungsmentalität sei das, und schließlich müsse der kleine Mann seine Lohnerhöhung auch erst erstreiken. Dümmer geht's nimmer.

Es ist längst überfällig, dass Abgeordnetendiäten automatisch angepasst werden, um dem regelmäßigen Ritual des öffentlichen Aufschreis zu entgehen. Ich bin gegen Heimlichtuerei, aber auch dagegen, dass die gut 600 wichtigsten Repräsentanten unserer Demokratie in den Dreck gezogen werden. ... Was Parlamentarier in der Hauptstadt und in ihren Wahlkreisen

24 Gilt das so pauschal aber gleichermaßen für Abgeordnete mit und ohne herausgehobene politische Funktionen? Für solche mit Wahlkreisen in oder um Berlin - und für solche, deren Wahlkreise im Badischen, in Oberbayern oder am Niederrhein liegen?

25 Zur Sicht des Bundestagspräsidenten, repräsentativ für einen großen Teil deutscher Parlamentarier, siehe etwa seine Interviews in der Märkischen Allgemeinen vom 2. April 2013, S. POL2 („Die gesellschaftliche Balance ist aus den Fugen. Bundestagspräsident Lammert über hohe Gehälter und parlamentarische Höhenflüge") und in der Leipziger Volkszeitung vom 2. April 2013, S. 2 („Verdacht der Selbstbedienung reduzieren“).

$26 \mathrm{Zu}$ Abgeordneten und ihrer Repräsentationsleistung siehe neuerdings auch Oscar W. Gabriel, Repräsentationsschwächen und die zweite Transformation der Demokratie: Wer will in Deutschland direkte Demokratie?, in: ZParl, 44. Jg. (2013), H. 3, S. 592 - 612; Sven T. Siefken, Repräsentation vor Ort: Selbstverständnis und Verhalten von Bundestagsabgeordneten bei der Wahlkreisarbeit, in: ZParl, 44. Jg. (2013), H. 3, S. 486 - 506; Suzanne S. Schüttemeyer, Abgeordnete: Repräsentanten oder Rädchen im Getriebe?, in: Heinrich Oberreuter (Hrsg.), Macht und Ohnmacht der Parlamente, Baden-Baden 2013, S. 59 - 78. 
leisten, das geht weit über die übliche tarifliche Arbeitszeit normaler Arbeitnehmer hinaus. Also Schluss mit Shitstorms und Bashing!

Politiker sollen ein angemessenes Gehalt bekommen, sonst haben wir nur noch Beamte und Funktionäre im Parlament. ${ }^{27}$... Doch dann sollte man sie ihre Arbeit machen lassen und sie so behandeln, wie man selbst behandelt werden möchte. Schließlich sind sie Volksvertreter und keine Räuberbande."

Typische Reaktionen von Lesern hierauf, abgedruckt in der Bild am Sonntag eine Woche später, lasen sich so:

„Ihr Loblied auf die Abgeordneten spottet jeder Beschreibung. Ohne einen Cent zu zahlen, erhalten diese Herrschaften eine Altersversorgung, über [sic] deren Höhe die Masse der Rentner nur träumen kann. Also: Ihre Äußerung, dass Deutschland die beste Demokratie der Welt hat, schreit zum Himmel!“

„Ich bin erstaunt, wie vehement sie unsere ,armen Politiker ${ }^{`}$ in Schutz nehmen. Eine jährliche Erhöhung der Bezüge ${ }^{28}$ ist für den normalen Bürger, Rentner, Hartz-IV-Empfänger und viele mehr ein Schlag ins Gesicht."

Auch Peter Hahne fiel angesichts solcher Reaktionen auf, was jeden im Bereich politischer Bildungsarbeit Tätigen ohnehin nicht überrascht: Bei alledem geht es nicht um rationales Urteilen, sondern um freien Lauf für verbreiteten Ärger über die politische Klasse. Solcher Zorn aber entzündet sich nur am Politikereinkommen, bezieht seinen Brennstoff hingegen aus viel tieferen Schichten der deutschen Politischen Kultur. Auf sie ließ auch Hahne blicken, als er in seiner nächsten Bild-Kolumne am Folgesonntag seine Erfahrungen mit der Leserpost wie folgt beschrieb:

„Beinahe jede zweite Leserzuschrift ... begann mit dem Satz: ,Bisher haben wir Ihre Gedanken gern gelesen, doch jetzt ... . Was hatte ich falsch gemacht, hatte ich Verbrecher verteidigt? Ich hatte mir nur erlaubt, für eine gerechte Bezahlung unserer Parlamentarier zu plädieren und davor gewarnt, die Abgeordneten als höchsten Souverän unserer Demokratie ${ }^{29}$ dauernd in den Dreck zu ziehen.

Wie dramatisch muss es um das Ansehen unserer Politiker bestellt sein, dass mir Leser deshalb die Treue kündigen! An einen solchen Empörungs-Tsunami kann ich mich in fast zwei Kolumnenjahrzehnten nicht erinnern. ... nur wenige Leser waren meiner Meinung, die meisten spielten ,Haut den Lukas'. ... Wenn ich die Meinung unserer Leser zusammenfasse, wundere ich mich nicht mehr, dass die größte Volkspartei inzwischen die der Nichtwähler ist. Das Ansehen unserer Politiker ist im Keller, und zwar so tief, wie ich es im Traum nicht geahnt hätte.

Da es ja nicht um das Ansehen einer kleinen Klitsche, sondern um den Bestand unserer Demokratie geht: Die Politiker müssen alles daransetzen, den (Vor-)Urteilen der Bürger offen zu

27 Ähnlich argumentiert auch Dorothea Siems in ihrem Kommentar „Politik als Beruf“, in: Die Welt vom 3. April 2013, S. 3. Zu dieser Thematik siehe im Einzelnen aber unten den Abschnitt 4.7.

28 Tatsächlich können fortan die - gemäß den Kommissionsempfehlungen - an die Nettolohnentwicklung gekoppelten Abgeordnetenbezüge auch sinken.

29 Das ist allerdings eine schiefe Formulierung. Nicht einmal das Parlament ist in Deutschland souverän, also über der Verfassung stehend - und schon gar nicht sind das „die Abgeordneten“. Man kann sogar mit guten Gründen die These vertreten, im Verfassungsstaat gäbe es überhaupt keinen Souverän mehr, also gar keinen Inhaber einer höchsten und nicht von anderen Institutionen kontrollierten Macht; siehe etwa Martin Kriele, Einführung in die Staatslehre. Die geschichtlichen Legitimitätsgrundlagen des demokratischen Verfassungsstaates, Opladen 1994. 
begegnen. ${ }^{30}$ Nur sie haben es in der Hand, dass ihnen Achtung und nicht Verachtung entgegengebracht wird. Wer vorgibt, das Volk zu vertreten ${ }^{31}$, kann nicht zur Tagesordnung übergehen, wenn er vom Volk verbal derart getreten wird.“

„Zur Tagesordnung übergehen“ sollte da ganz gewiss niemand, allerdings auch nicht die Journalistenschaft als zentrale Verbreiterin und Verstärkerin, doch auch als - dem immer wieder unter Beweis gestellten Potential nach: durchaus chancenreiche - Bekämpferin entsprechender (Vor-) Urteile. Populärer Ärger sitzt hier ganz tief - und richtet sich nicht nur gegen eine gute Bezahlung, sondern oft schon gegen die Existenz von (zumindest: so vielen) Abgeordneten. In der Berliner Morgenpost vom 4. April 2013 meinte beispielsweise ein Leserbriefschreiber:

„Wenn wir dann [nach Begrenzung der Abgeordneteneinkommen] tatsächlich nicht mehr genug Abgeordnete finden, ist dies umso besser, denn das Vorhaben, die Anzahl der Abgeordneten zu begrenzen, hat ja bislang auf anderem Wege noch nicht geklappt. Im Gegenteil: Wegen der Überhangmandate wurde die Zahl der Abgeordneten sogar noch erhöht."

Kraut und Rüben dieser Art verlegen den Weg zur Erkenntnis der einschlägigen Zusammenhänge. Doch gerade deshalb gilt es, solche Kritik sorgfältig aufzunehmen, an ihr das Triftige vom Irrigen zu unterscheiden - und beim Triftigen dann das politische System, beim Irrigen den Bildungsstand der Bevölkerung zu verbessern. Den richtigen Schritt tat in dieser Hinsicht Heribert Prantl in der Süddeutschen Zeitung vom 3. April 2013.32 Er argumentierte:

„Der nun ... vorgelegte Expertenvorschlag, die Höhe der Diäten am Grundgehalt von Richtern zu orientieren und Erhöhungen an den Nominallohnindex zu knüpfen, ist ... zwar nicht der Weisheit letzter Schluss, aber vernünftig - weil er, hoffentlich, einen plausiblen Maßstab und ein wenig Ruhe in eine demokratiegefährdende Debatte bringt, ... [die] seit Jahrzehnten

30 Warum eigentlich nur die Politiker? Wieso nicht auch Journalisten, die dann - wie gerade das Beispiel Peter Hahnes zeigt - freilich sturmfeste Bekleidung und Durchhaltekraft bräuchten? Der Bundespräsident hat diesbezüglich, wie es auch seines Amtes ist, bei seinem Antrittsbesuch in Hannover einen richtigen Beitrag geleistet, indem er mehr Respekt vor Politikern forderte: „Viele Menschen im Land mögen Regierende nicht, aber wenn es keine Regierenden gäbe, würde das Chaos einziehen. ... Und deshalb braucht die ganze Gesellschaft einen vernünftigen Zugang zu einer Kultur der Achtung vor denen, die sich die Mühe machen, öffentlich Verantwortung zu tragen" (zitiert nach FAZ vom 12. April 2013, S. 4).

31 Hier endet anscheinend Hahnes Widerstand gegen auf ihn einstürmende Leservorurteile: Aus Abgeordneten werden „,vorgebliche Volksvertreter“.

32 In die falsche Richtung geht allerdings Prantls im gleichen Artikel geäußerter Vorschlag, die Kostenpauschale - wie inzwischen etwa in Nordrhein-Westfalen - in die Diäten zu integrieren. Bei Abgeordneten im großstädtischen Bereich mit geringen Fahrtkosten und keinem Bedarf an mehr als einem einzigen Wahlkreisbüro wird dann nämlich einfach das auch privat verfügbare Gehalt größer sein, während Abgeordnete in ausgedehnten Wahlkreisen einen nennenswerten Teil ihres Gehalts wirklich für die Wahlkreisarbeit brauchen. Vor diesem Hintergrund ist es besonders merkwürdig, wenn - wie von Karl Doemens, „Lammerts Diätenreform“, a.a.O. (Fn. 17), derlei ausgerechnet unter dem Feldzeichen der Gerechtigkeit (!) gefordert wird. Zielführender ist da schon der von Hans Herbert von Arnim unterstützte Vorschlag, einen im Vergleich zu heute klar geringeren, für alle Abgeordneten typischen, Betrag pauschal zu erstatten, doch alle Auslagen, die aufgrund von Besonderheiten des Wahlkreises entstehen (mehr als ein Wahlkreisbüro, zweiter Wohnsitz am Parlamentsort usw.), bis zu einer bestimmten Obergrenze nur anhand präziser Nachweise erstattet zu bekommen (siehe sein Interview „Rosinenpicken für Abgeordnete“, in: FR vom 15. April 2013, S. 6). Derlei wurde von der Kommission zwar durchaus erwogen, doch ohne abschließende Einigung; siehe Bericht und Empfehlungen, in: Suzanne S. Schüttemeyer I Edzard Schmidt-Jortzig (Hrsg.), a.a.O. (Fn. 1), S. 89 - 91. 
in ziemlich unangemessener, ja in frivol demokratieschädlicher Weise geführt [wird]. Immer dann, wenn in einem Parlament über die Gehälter der Abgeordneten beraten wird, beginnt eine öffentliche Diskussion, die so tut, als würde jetzt die ungerechtfertigte Bereicherung legalisiert und als würden sich die Volksvertreter nur deshalb vom Volk wählen lassen, um im Parlament leichtes Geld zu verdienen.“

Wie Peter Hahne erkennt auch Heribert Prantl „ein abgründiges Misstrauen an der Integrität der Volksvertreter, an dem diese aber nur zu einem kleinen Teil selber schuld sind“. Und dann formulierte er aufs schönste jenes Dilemma, aus dem der Kommissionsvorschlag endlich einen Ausweg weist:

„Schuld an der Giftigkeit der Diätendebatte ist unter anderem ... das Grundgesetz. Dort steht ... die Vorschrift, dass ... über die genaue Höhe der Diäten per Gesetz entschieden werden muss. Das beißt sich aber mit dem Prinzip, dass niemand Richter in eigener Sache sein soll; und das ist ein Satz, der tief im kollektiven Rechtsbewusstsein verankert ist. Aus diesem Rechtsbewusstsein erwächst daher das Unbehagen darüber, dass sich die Abgeordneten ihre Diäten selbst genehmigen. ... Diesem Dilemma entkäme man mit einer Grundgesetzänderung, die das Parlament von der Festsetzung der Diäten befreit - und diese komplett auf ein externes Gremium oder auf einen ins Grundgesetz zu schreibenden Automatismus überträgt. ${ }^{33}$ Eine solche Grundgesetzänderung ist aber unrealistisch.“

Der Kommissionsvorschlag - Koppelung eines Grundgehalts an die Besoldung der Bundesrichter und weitere Gehaltsentwicklung parallel zum Nettolohnniveau - geht tatsächlich gerade so weit in die von Prantl empfohlene Richtung, wie das ohne Grundgesetzänderung sowie angesichts des Verfassungsgerichtsurteils von 1975 möglich ist. Offen ist, ob die Parlamentarier zu Beginn der nächsten Wahlperioden immer noch den Mut haben werden, auf diesem Weg weiterzugehen - denn nach allen Erfahrungen aus der Vergangenheit wird sich jeweils ein Sturm öffentlicher Empörung über derlei „Selbstbedienung auf Vorrat“ erheben, der - wohl schon während der jeweiligen Koalitionsverhandlungen - parlamentarische Standfestigkeit erproben wird.

\section{Die zentralen Kritikpunkte in Tagespresse und Bürgerreaktionen}

\section{1. „Ein Gefälligkeitsgutachten!“}

Alle Gemeinplätze der Parlamentarismuskritik bespielt in Deutschland traditionell Hans Herbert von Arnim. So hielt er es auch anlässlich des Berichts der Expertenkommission. ${ }^{34}$

33 In Thüringen steht eine entsprechende Indexregelung in Art. 54 Abs. 2 der Verfassung und wurde 1998 vom Thüringer Verfassungsgerichtshof anlässlich einer vom Bund der Steuerzahler inspirierten Klage der PDS-Landtagsfraktion bestätigt. Gleichwohl hat auch in Thüringen die Empörung über regelmäßige Diätenanpassungen nicht abgenommen. Trotzdem hoffte im Jahr 2013 der damalige Bundestagsvizepräsident Hermann Otto Solms, „wenn ein solcher Mechanismus [erst einmal] akzeptiert ist, wird er keine große Diskussionen nach sich ziehen“" (AFP-Meldung vom 2. April 2013). Vor solchen Hoffnungen warnte indes Bernhard Walker, Der Bundestag sollte Diäten endlich auf Eigenvorsorge umstellen, in: Badische Zeitung vom 3. April, S. 4: Es „sollte sich der Bundestag nicht der Illusion hingeben, dass ein quasi automatisches Verfahren den Stammtisch-Zorn besänftigt. Wer stumpfsinnig genug ist, Abgeordnete per se faul und bestechlich zu finden, wird auch keinen Index gutheißen".

34 Die folgenden Zitate entstammen Hans Herbert von Arnim, a.a.O. (Fn. 32). 
Nicht nur sind von Arnim die von der Kommission vorgeschlagenen Abgeordnetenbezüge zu hoch, zumal er sie für ganz zu Unrecht am Einkommen von Bundesrichtern orientiert hält. Diese erhielten nämlich keine steuerfreie Kostenpauschale ${ }^{35}$, hätten eine weniger günstige Altersversorgung ${ }^{36}$ und dürften keinen Nebenberuf ausüben. Auch die künftige „automatische“ Anpassung der Diäten missbilligt er, denn: „Mit dieser Dynamisierung wird die öffentliche Kontrolle ausgeschaltet. Das Verfassungsgericht hat ausdrücklich verlangt, dass jede einzelne Erhöhung in einem parlamentarischen Prozess beschlossen wird.“ Das ist eine tatsächlich über lange Zeit akzeptierte Interpretation, aus der die Expertenkommission mit guten Gründen einen Ausweg aufzuzeigen versucht hat. Doch solche Befreiungsabsichten nimmt von Arnim der Kommission besonders übel - und zwar nicht nur beim vorgeschlagenen „Automatismus“ der Gehaltsanpassung, sondern auch bei der Einführung weiterer Zulagen für die Inhaber herausgehobener Parlaments- und Fraktionspositionen. Besonders wurmt ihn, dass der Gesetzgeber hier das Verfassungsgericht gleichsam austricksen könne ${ }^{37}$ :

„Die Kommission weiß genau, dass vermutlich doch niemand das Bundesverfassungsgericht anrufen wird. Politiker, die von den Vorschlägen profitieren, werden nicht klagen, und die Bürger haben kein Klagerecht. Von sich aus darf das Gericht aber nicht tätig werden. "38

Hans Herbert von Arnims grundlegender Vorwurf aber geht dahin, dass die aus (ehemaligen) Parlamentariern „und anderen parlamentsnahen Personen“ zusammengesetzte Kommission „einen extrem einseitigen Bericht vorgelegt [habe]. Letztlich ist das ein Gefälligkeitsgutachten“, bei dem die Kommission „völlig einseitig wie ein Interessenvertreter nur die Argumente aufgelistet [hat], die für ihre Klientel sprechen" ${ }^{39}$. Also betreibe die Kommission „Rosinenpickerei“. Sie wolle einfach „die Entschädigung der Parlamentarier anheben, alle problematischen Vorteile der Abgeordneten segnet sie dagegen ab“.

Selbst wer ein Körnchen Wahrheit in von Arnims Position sehen will, tut gut daran, auf drei Dinge zu achten. Erstens waren unter den elf Kommissionsmitgliedern zwar vier ehemalige Abgeordnete, ein ehemaliger Direktor beim Deutschen Bundestag sowie eine Hochschullehrerin, die zu den führenden deutschen Parlamentarismusexperten zählt. Doch hätte eine ohne Parlamentarier und Parlamentarismusexperten auskommende Kommission wirklich mehr Sachkunde und Fachautorität haben können? Und müssen die übrigen Kommissionsmitglieder - ein ehemaliger Bundesverfassungsrichter, ein Landesverfassungsrichter

35 Sie haben allerdings auch keinen Aufwand für die Betreuung eines Wahlkreises.

36 Was offenkundig ganz wesentlich damit zu tun hat, dass sie kein Amt auf Zeit ausüben, das mitunter in recht unübliche Erwerbsbiographien eingebettet ist, und dass bei den Bundesrichtern auch niemand verlangt, ihr Amt solle - um der Demokratie willen - ungeachtet von Herkunft und bisherigem Lebenslauf jedem ohne Sorge um seine Alterssicherung zugänglich sein.

37 Siehe hierzu auch Hans Herbert von Armin, Eine Kriegserklärung an das BVerfG. Zum Bericht der Schmidt-Jortzig-Kommission, in: Neue Zeitschrift für Verwaltungsrecht, 32 Jg. (2013), H. 8, S. $1-11$.

$38 \mathrm{Ob}$ von Arnim unter solchem Tätigwerden wohl auch weitreichende obiter dicta des Gerichts verstehen wollte, wie sie beim Diätenurteil von 1975 aus einer zunächst nur ein Landesparlament betreffenden Entscheidung anschließend eine Verfassungspraxis mit 40 Jahre lang delegitimierender Wirkung machten?

39 Das ist auch der Vorwurf, den Barbara John im Tagesspiegel vom 21. April 2013, S. 6 unter dem (Unter-) Titel erhebt: „Über Volksvertreter, die sich nicht selbst bedienen wollen - und es trotzdem tun". 
und Professor für Sozialrecht, zwei Rechtsanwälte mit engen Wirtschaftsverbindungen und eine Professorin für Öffentliches Recht - einfach deshalb als „zugunsten von Abgeordneten befangen" gelten, weil sie besondere Kenntnisse zu deren Aufgabe, deren Besoldung und Alterssicherung aufweisen? Zweitens ist doch nicht zu übersehen, dass die Logik der Altersversorgung von Abgeordneten nach den Kommissionsvorschlägen der gesetzlichen Rentenversicherung angeglichen wird, was schwerlich in eine falsche Richtung zielen kann. Und drittens wollte die Kommission nicht für den Papierkorb arbeiten, sondern zu einem greifbaren Ergebnis beitragen. Das aber geht gerade in einer parlamentarischen Demokratie nicht ohne die Bereitschaft, sich auch auf die Sichtweisen und Interessen von Parlamentariern einzulassen. Aus allen diesen Gründen geht von Arnims Behauptung entschieden zu weit, der Kommissionsbericht sei auch noch „ein Anschlag auf die politische Kultur dieses Landes“. Eher ist es so, dass die Reaktionen auf den Kommissionsbericht Problemzonen unserer Politischen Kultur sichtbar machen.

\section{2. „Ein Ja zur Selbstbedienungsmentalität!“}

Ein Kernpunkt der Kritik am Parlament ist die regelmäßig behauptete „Selbstbedienungsmentalität" der Abgeordneten. Tatsächlich vergeht kaum ein Jahr, in dem es die Diskussion in einem unserer 17 Parlamente um eine Anpassung - bislang: stets Erhöhung - der Abgeordnetenbezüge nicht in die Wahrnehmung der Öffentlichkeit schafft. Viel weniger Durchschlagskraft hat die Mitteilung, das Bundesverfassungsgericht selbst habe 1975 in einem wie es der Kommission schien: allzu willfährig aufgefassten - Urteil den Parlamenten auferlegt, selbst über die Abgeordnetengehälter zu entscheiden. Davon nichts wissend, kommen viele Bürger zu Unmutsbekundungen wie den folgenden: „Die Selbstverständlichkeit, mit der sich die Abgeordneten eine Gehaltserhöhung genehmigen, ist schon erstaunlich“, oder: „Wenn Menschen die Möglichkeit gegeben wird, über ihr eigenes Einkommen zu entscheiden, siegt die Gier." 40 Viel besser traf allerdings Daniel Friedrich Sturm die Sache, als er in der „Welt“ vom 3. April 2013 unter dem Titel „Gehaltsdebatte mit PeinlichkeitsPotenzial“ formulierte:

„Viele Abgeordnete sind es leid, in eigener Sache zu entscheiden. Die teilweise bescheidenen Diätenerhöhungen [der Vergangenheit], in Zahlen für jedermann vorstellbar, lösen stets mehr öffentlichen Groll aus als andere staatliche Ausgaben. ... Um die als leidig wahrgenommene, wiederkehrende öffentliche Debatte über die Höhe zu vermeiden, schlägt die Kommission geradezu einen Automatismus vor: ..."

Diese „Alternative zur Selbstbedienung“ kam aber auch nicht gut an. Bild vom 20. März 2013 titelte etwa: „Abgeordnete sollen jedes Jahr automatisch mehr Geld kriegen“ - und das klang, trotz des neutralen Folgetextes, ganz wie ein Vorwurf. In der Frankfurter Allgemeinen Zeitung konnte man am 4. April 2013 unter der Überschrift „Im Sog der Beamten?" sogar die Behauptung lesen, dass die Abgeordneten gerade (!) bei einer „automati-

40 Zitiert aus Annett Meiritz, Politikergehälter: Diätendebatte entzweit Unionsfraktion, in: Spiegel Online vom 2. April 2013, http://www.spiegel.de/politik/deutschland/politikergehaelter-unionsfraktion-will-keine-eilige-diaetenerhoehung-a-892023.html (Abruf am 4. Juni 2014), sowie aus einem Leserbrief im Artikel von Mathias Stengel, „Bezüge an Rentenniveau koppeln“, in: Berliner Morgenpost vom 4. April 2013, S. 25. 
schen Diätenanpassung“ - und eben nicht (!) bei „Entscheidungen in eigener Sache“ - ihre Glaubwürdigkeit verlören:

„Parlamentarier sind keine Beamte. Daher müssen sie weiterhin und immer aufs Neue über ihre Einkünfte entscheiden. Durch einen Automatismus verlören sie auch an Glaubwürdigkeit, weil sie bei Besoldungsgesetzen über sich selbst mitentschieden."

Warum das - wenn es denn ein tatsächlich wichtiger Wirkungszusammenhang wäre - der Glaubwürdigkeit von Parlamentariern noch mehr Abbruch täte, als wenn sie in sozusagen „dreister Offenheit“ das eigene Gehalt festlegen, sagte der Autor allerdings nicht. Doch er legte nahe, dass in den Augen der Öffentlichkeit wohl nur Abgeordnete mit einem „eingefrorenen" - und eines Tages dann eher symbolischen - Gehalt akzeptabel wären. Damit wäre fast wieder die Lage vor der Einführung von Diäten im Jahr 1906 erreicht $^{41}$, und es stellten sich alle Probleme einer so bewirkten sozialen Exklusivität der Abgeordnetenschaft aufs Neue.

Also zeigt sich in der Kritik sowohl an der bisherigen als auch an der neuen Regelung der Abgeordnetenbesoldung ein schlechterdings nicht zu beseitigendes Dilemma: „Automatische" Gehaltsanpassungen gelten als schlecht, Entscheidungen von Parlamentariern über ihr Gehalt aber auch ${ }^{42}$; und wenn dann eben eine externe Kommission ein höheres Gehalt vorschlägt, so wird diese Kommission entweder als „nicht unabhängig genug“ oder als „zu parlamentsfreundlich “ 43 kritisiert - oder es wird dem Parlament vorgeworfen, von ihm selbst zu tragende Verantwortung delegiert zu haben. Im Hintergrund steht, eher atmosphärisch als unmittelbar zu fassen, ein Vorwurf dahingehend, dass Abgeordnete überhaupt Gehalt bekommen - und dann auch gar ein höheres als der Bevölkerungsdurchschnitt. ${ }^{44}$ In der Berliner Morgenpost vom 4. April 2013 formulierte beispielsweise ein Leserbriefschreiber:

41 Zur damaligen Diskussion siehe auch Philipp Austermann, a.a.O. (Fn. 18), S. 117 - 122.

42 „Bisher legen die Abgeordneten ihre Diäten selbst fest“, schrieb die SZ - eine DPA-Meldung wiedergebend - am 20. März 2013, S. 6, wobei dieser sachliche Satz in einem ganz sachlichen Artikel dennoch wie ein Vorwurf klang.

43 Siehe etwa die folgenden Online-Kommentare auf der Webseite der Zeitung Die Welt zur Berichterstattung über den Abschlussbericht der Kommission vom 21. März 2013: „Also ein unabhängiger Experten Rat aus Politikern / ehemaligen politikern bestimmt für sich selbst was Sie den im alter bekommen, das will ich auch!“ (Rechtschreibfehler im Original). Oder: „Ja wer hat denn hier geglaubt, dass es Frösche gibt, die selbst freiwillig ihren -Teich trockenlegen??? Das gabs noch nie - und in diesem Punkt sind sich immer alle einig. Nach dem Motto: ,Ich kenne keine Parteien mehr - sondern nur noch Absahner! ““

44 Gleiches gilt auch für die Altersversorgung, woraus sich dann folgender Reformvorschlag für die Altersversorgung der Bevölkerung ableitet: „Bei der nächsten Rentenreform wird eine Kommission eingesetzt, die ausschließlich aus Rentenbeziehern besteht. Die Zusammensetzung richtet sich nach dem Anteil der Rentenbezieher: Niedrigrentner, Durchschnittsrentner, Besserrentner. Das Ergebnis wird sicherlich alle Rentner richtig freuen" (Online-Kommentar auf der Webseite der Zeitung Die Welt zur Berichterstattung über den Abschlussbericht der Kommission vom 21. März 2013). Und die empfundene Verletzung von Fairness- oder Gerechtigkeitsstandards bei der Festsetzung von Abgeordnetengehältern ist offenbar auch der Argumentationshintergrund des folgenden Vorschlags in einem Leserbrief, abgedruckt in der Berliner Morgenpost vom 4. April 2013 (Mathias Stengel, a.a.O. (Fn. 40)): Der Bundestagspräsident habe es wieder einmal eilig mit einer Diätenerhöhung, wobei die Abgeordneten ohnehin schon „zu den Spitzenverdienern in Deutschland“ gehörten; und deshalb wäre es schön, „wenn sich der Bundestagspräsident [auch] für die Einführung eines armutsfreien Mindestlohns in Deutschland noch vor der Bundestagswahl ... stark gemacht hätte“. 
„Die Bezahlung der Abgeordneten ist jetzt schon zu hoch. Bis ein normaler Richter Bundesrichter wird, dauert es Jahrzehnte. Also: Weg mit der Selbstbedienung der Bundestagsabgeordneten und vor allem mit der Steuerbefreiung und her mit einem Gesetz, das die Bezüge der Volksvertreter begrenzt."

Vor solchem Hintergrund kann es kaum anders denn als Provokation wirken, wenn die Kommission auch noch ausführlich Gründe dafür angibt, warum Abgeordnete bedeutend mehr als der Bevölkerungsdurchschnitt verdienen sollten.

\section{3. „Zu viel Geld für zu wenig Leistung!“}

„Wie viel sind dem Volk seine Volksvertreter wert?" - so formulierte der Berliner Kurier die zentrale Frage in einem Artikel vom 3. April 2013 mit dem Titel „9,6\% mehr Gehalt: Dreist oder gerecht?“. Die demoskopischen Befunde hierzu sind eindeutig. 2008 hielten laut einer Forsa-Umfrage 84 Prozent der Deutschen eine Diätenerhöhung bis 2010 um 16 Prozent für zu hoch. Zugleich stimmten nur zehn Prozent der Aussage zu, ein Bundestagsabgeordneter sei sein Geld wert; 52 Prozent stimmten dem (gar) nicht zu, 14 Prozent waren unsicher und 26 Prozent sagten einfach „weder-noch“45. Anfang April 2013 waren es bei einer Emnid-Umfrage für den Focus 86 Prozent, die höhere Bezüge für die Bundestagsabgeordneten ablehnten, während nur neun Prozent meinten, deren Einkommen solle steigen.

Welche Einschätzungen stehen hinter solchen Antworten? Allein auf die persönliche Amtsführung hob die Antwort von Gregor Mayntz in der Rheinischen Post vom 2. April 2013 ab: „Natürlich sind Abgeordnete [bereits] mit 8.252 Euro überbezahlt, wenn sie den Job nur mit der linken Hand erledigen und mit der rechten eifrig hinzuverdienen. Aber die meisten kämpfen sich durch eine 90-Stunden-Woche, sind fleißig und wichtig wie diejenigen, die viel mehr verdienen." Auf die Gesamtstruktur unseres Parlamentarismus hingegen zielte der folgende Leserbrief aus der Berliner Morgenpost vom 4. April 2013, in dem so ziemlich alle Stereotypen populärer Parlamentswahrnehmung vorkommen:

„Warum und weshalb eine Erhöhung der Diäten? Die meisten Entscheidungen werden doch in Brüssel getroffen. Bei den Entscheidungen im Parlament darf der Abgeordnete ja doch nur die von den Fraktionsspitzen schon zuvor getroffenen Entscheidungen tragen. Es sind hoch bezahlte Marionetten. ${ }^{46}$ Es gibt nur wenige Ausnahmen, wo Politiker von der Fraktionsmeinung abweichen. Sieht man die oft geringe Anwesenheit der Abgeordneten bei Parlamentssitzungen, dann fragt man sich, wo sind die denn? Ich sehe jetzt nicht den richtigen Zeitpunkt $^{47}$ für die kräftige Erhöhung der Diäten!“

Zwar hat sich die Expertenkommission in ihrem „Leitbild“ viel Mühe gegeben, sowohl die Wichtigkeit von Parlamentariern als auch die Herausforderungen ihres Berufes so klar zu schildern, dass eine überdurchschnittliche Entlohnung von Abgeordneten sowohl vielen Journalisten als auch deren Lesern einleuchten könnte. Doch diese Rechnung ging nicht

45 Quelle: BITKOM. Harris Interactive, 2014.

46 Ein Leserbriefschreiber aus der Zeitung Die Welt vom 5. April 2013, S. 2, schränkt dieses Argument wenigstens so ein: „Der über die Liste eingerückte Parlamentarier hat sich indes den Wünschen der Fraktionsführungen zu beugen. Stemmt er sich dagegen, ist der sichere Listenplatz bei der nächsten Wahl unter Umständen gefährdet.“

47 Geht man nach Volkes Stimme und deren Echo in den Reihen der jeweiligen parlamentarischen Opposition, dürfte niemals ein ,richtiger Zeitpunkt“ kommen. 
auf. Weniger systematisch als vielmehr ironisch hielt es nämlich so mancher Journalist mit dem „Leitbild“. Annett Meiritz etwa schrieb in ihrem Artikel „Schlaflos, gestresst, unterbezahlt" in Spiegel Online vom 4. April 2013, im Analyseteil des Kommissionsberichts zum Beruf des Abgeordneten lese man ...

„eine Beschreibung des härtesten Jobprofils Deutschlands. Je länger man sich darin vergräbt, desto bedauernswerter erscheint der Alltag des Parlamentariers. Kurz gesagt: Dagegen sind Jobs wie Tatortreiniger oder Fließbandarbeiter Traumberufe“.

Nicht anders schätzte das Fabian Leber im Tagesspiegel vom 8. April 2013 ein: Die Kommission zeichne „ein Bild der Parlamentariertätigkeit, das den einfachen Bürger in Sack und Asche gehen lässt“, und es stelle sich „die Frage, ob den Parlamentariern mit dieser Lobhudelei ein Gefallen getan wurde“. Eigentlich solle es nur um eine Diätenreform gehen - doch es tue „die Kommission so, als brauche man im Parlamentsalltag inzwischen Supermann-Qualitäten“.

Tatsächlich wird man einen zwingenden Zusammenhang zwischen den Anforderungen an einen Abgeordneten und der Höhe des ihm zu zahlenden Gehalts schwerlich finden. Immerhin gab es auch zu Zeiten viel geringerer Diäten keinerlei anhaltende Schwierigkeiten, Bewerber für Parlamentsmandate zu finden. Ganz offensichtlich ist es beim Politikerberuf nicht Geld allein, was anzieht und Berufszufriedenheit stiftet. Zu Recht weist Meiritz im oben zitierten Artikel darauf hin, dass im Kommissionsbericht „die Vorteile, mit denen der politisch interessierte Nachwuchs aufwächst, überhaupt nicht thematisiert werden. Die neue Netzöffentlichkeit des politischen Betriebs wird zum Beispiel mehr als Gefahr denn als Chance gesehen. Moderne Kommunikationsformen ... all das bewertet die Kommission überwiegend als Mehrbelastung" - und nicht als Teil des immateriellen Reizes des Abgeordnetenmandats. Zu Recht also fügt Fabian Leber im Tagesspiegel vom 8. April 2013 dem die Beobachtung hinzu:

„Dass der Abgeordnetenberuf wegen der [inzwischen allzu geringen] Höhe der Diäten unattraktiv geworden ist, dafür gibt es keine Belege. Es wird auch in Zukunft genügend Menschen geben, die es genießen, bei allen möglichen Anlässen nach ihrer Meinung gefragt zu werden.“

Tatsächlich ist es bei Politikern ebenso wie bei Künstlern oder Wissenschaftlern: Nur des Geldes wegen lohnt ein solcher Beruf nicht; sondern was anzieht, ist vor allem jene Lebenswelt, zu der er Zugang gewährt, und das Gestaltungspotential, das er erschließt. Das also muss man ebenfalls auf die Rechnung setzen, wenn man über Gerechtigkeitsmaßstäbe bei der Festsetzung von Politikereinkommen nachdenkt.

\section{4. „Privilegien bei der Altersversorgung!“}

Nicht wenig Kritik zog auch der Verzicht der Expertenkommission auf einen gemeinsamen Veränderungsvorschlag zur Regelung der Altersversorgung auf sich. Die „Welt“ vom 21. März 2013 widmete dem sogar einen eigenen Artikel $^{48}$, der sich weitgehend die entsprechende Kritik des Bundes der Steuerzahler zu eigen und diese Kritik somit den Lesern mit

48 Martin Greive / Dorothea Siems, Altersversorgungspläne für Abgeordnete in der Kritik, in: Welt Online vom 21. März 2013, http://www.welt.de/politik/deutschland/article114623784/Altersversorgungsplaene-fuer-Abgeordnete-in-der-Kritik.html (Abruf am 4. Juni 2014). Auf diesen Artikel beziehen sich oben und nachstehend zitierte Online-Kommentare von Lesern der "Welt“. 
Nachdruck bekannt machte. Der Tenor: Gerade bei der Altersversorgung gelte es, Privilegien abzubauen, weshalb es ein großer Fehler, eine verpasste Chance wäre, dass die Kommission hier alles so belassen wolle, wie es derzeit sei. ${ }^{49}$

Mit den Einzelheiten dieser durchaus vertrackten Thematik befasste sich die über Massenmedien und Blogs verbreitete Kritik allerdings kaum. Auch Berechnungsbeispiele sucht man vergeblich, die - im Vergleich zu anderen verantwortungsvollen Berufen - zeigten, wie viel ein derzeit mit durchschnittlich 44 Jahren in den Bundestag einziehender Abgeordneter nach einer durchschnittlichen parlamentarischen Verweildauer von zehn Jahren ab dem Renteneintrittsalter (im Durchschnitt gut zehn Jahre nach dem Mandatsende erreicht), monatlich an aus dem Mandat erworbenen Versorgungsansprüchen beziehen wird. Ohne solche Zahlen aber bleibt die Rede von „für den Normalbürger unerreichbar hohen“ Altersbezügen ganz im Bereich von Vermutungen und Vorurteilen - zumal dann, wenn statt der Durchschnittswerte die bei jahrzehntelanger Parlamentszugehörigkeit theoretisch erreichbaren Maximalwerte der Altersversorgung berichtet werden. Im Übrigen fehlen in der massenmedialen Berichterstattung konkrete Hinweise darauf, weshalb sich die Kommission tatsächlich mit einer klaren Reformempfehlung so schwer tat - außer der Unterstellung, sie habe, parteiisch zusammengesetzt, die Abgeordneten einfach hätscheln wollen. Dabei hätte man sich doch leicht mit den ausführlich im Kommissionsbericht dargelegten Argumenten und Gegenargumenten befassen können.

Dies versäumend, ließ die Presse viele Bürger mit ihren Vermutungen und Schnellurteilen allein. Typisch für diese ist der folgende Online-Kommentar vom 21. März 2013 zur Berichterstattung der „Welt“ über den Abschlussbericht der Expertenkommission:

„Die feinen Damen und Herren sollen gefälligst in die Rentenkasse einzahlen. Die Abgeordneten sind nichts Anderes als auf 4 Jahre bestellte Zeitarbeiter. ${ }^{50}$ Schluss mit den Luxuspensionen!“

Ein so klarer Ausdruck von Sozialneid geht vermutlich über das einfache Nicht-gönnenKönnen parlamentarisch erworbener Versorgungsansprüche hinaus und hat unausgesprochene, doch mitgedachte Kontexte. Zu ihnen scheint zu gehören, dass ein Parlamentsman-

49 Unfair ist diese Kritik insofern, als es in der Expertenkommission auch ganz andere Regelungsvorschläge gegeben hatte, diese aber keine Mehrheit fanden. Im Übrigen ignorierten die Kritiker so gut wie völlig die einhellig verabschiedeten Kriterien einer angemessenen Altersversorgung und machten es sich damit ihrerseits viel zu leicht. Gleichwohl hätte es der Kommission gut angestanden, sich „volkspädagogisch“ auch mit - immer wiederkehrenden - Einwendungen wie der folgenden zu befassen, die am 24. Februar - nach der Neuregelung der Diäten - auf http://www. freiewelt.net/diaten-diaten-10025344/ gepostet wurde: „Predigen nicht diese gleichen Politiker noch der allerkleinsten Verkäuferin, sie habe gefälligst privat für das Alter vorzusorgen? Allein die Erhöhung der Bezüge reicht doch aus, um monatlich ein nettes Sümmchen in gleich welche Art einer Versorgungskasse einzuzahlen. Und in der Tat: Wieso soll ein Abgeordneter als Beamter gelten? Er ist doch nur so lange Abgeordneter, wie er gewählt ist. Die Pensionsregelung ist das Allerärgerlichste, denn die Diäten bekommt er ja nur, solange er im Parlament sitzt, während die Pensionsansprüche weit in die Zukunft reichen“" (Schreibfehler im Original).

50 Hierauf sprang auf der gleichen Kommentarseite ein anderer so an: „Tja, das Thema Zeitarbeiter hat was für sich. Denn wenn für Bundestagsabgeordnete die Regeln gelten, die für normale Zeitarbeiter auch per Gesetz gelten, dann würde sich hier schnell etwas ändern." Tatsächlich lassen sich die um das Bild vom „Zeitarbeiter“ gelagerten Argumentationsfiguren nicht beiseiteschieben, meint doch parlamentarische Demokratie ganz wesentlich „Vergabe von Macht auf Zeit“. Allerdings sind die mit der „Zeitlichkeit“ von Parlamenten verbundenen Wirkungsketten durchaus komplizierter. 
dat zu erringen eben doch als mehr erkannt wird denn als bloßes Ergattern einer „Zeitarbeiterstelle“: Es geht unübersehbar um sozialen Aufstieg. Zwar gehört Aufwärtsmobilität zu einer freien Gesellschaft und ruft als solche keinerlei erkennbare Kritik hervor. Doch das Gesamtbild populärer Parlamentskritik, aus der in etlichen Fußnoten dieses Beitrags angegebenen Literatur leicht zu ersehen, legt den folgenden Deutungskontext nahe: Nicht wenige Bürger provoziert, dass sich dieser Aufstieg über die weithin verachteten Parteien vollzieht, und obendrein noch - im Wesentlichen - außerhalb effektiver Kontrolle der Wähler darüber, wer konkret ins Parlament einzieht.

Es ist ja eher eine ehrwürdige Juristenformel, dass „die Abgeordneten vom Volk gewählt“ würden, während tatsächlich, zumindest in Deutschland, das Volk am Wahltag den internen Personalentscheidungen der Parteien akklamiert. ${ }^{51}$ Die aber begünstigen professionelle Abgeordnetenkarrieren, die vielfach vom konkreten Wahlausgang unabhängig sind, weil sie sich vor allem innerparteilicher Netzwerkbildung verdanken. Und dass sich solche ungeliebte „parteipolitische Strippenzieherei“ auch noch statusmäßig und finanziell zum Besten der Betroffenen auswirkt, scheinen viele Bürger erst recht nicht zu mögen. Falls nun aber diese empirisch erst noch zu erhärtende - Vermutung stimmt, wäre immerhin ein Weg zur Verringerung solchen Sozialneids erkannt: Es dürften die Bürger solchen Abgeordneten viel eher ein gutes Einkommen in Gegenwart und Zukunft gönnen, die sie nicht einfach von den Parteien als faktisch unabwählbare Mandatsbewerber präsentiert bekommen, sondern auf deren Auswahl sie durch Vorwahlen für alle Parlamentsmandate selbst Einfluss nehmen können. ${ }^{52}$

\section{5. „... und die Nebeneinkünfte?“}

Mancher, der grundsätzlich für eine „ordentliche Bezahlung der Abgeordneten“ eintritt, um eben dadurch deren Unabhängigkeit zu stärken, problematisiert dann die weiterhin möglichen Verdienste aus Nebenaktivitäten der Parlamentarier. ${ }^{53}$ Neuregelungen für sie vorzuschlagen, war zwar nicht die Aufgabe der Expertenkommission; inhaltlich gibt es aber einen Zusammenhang. Ihn formulierte etwa Michael Sommer, der Vorsitzende des DGB:

51 Abgesehen vom seltenen Fall jenes Direktmandatars, der in einem „unsicheren“ Wahlkreis antritt und dennoch nicht ,auf der Landesliste abgesichert“ wurde. Im Ausnahmefall kann das gutgehen; siehe die 2002 erfolgreiche Kandidatur des von seiner Partei nicht auf einem aussichtsreichen Listenplatz aufgestellten Grünen-Abgeordneten Hans-Christian Ströbele in einem - grünenfreundlichen - Berliner Bundestagswahlkreis.

52 Die Regeln entsprechender Vorwahlen für alle Landtags- und Bundestagsmandate, durchzuführen auf der Ebene von Wahlkreisen, könnten wie folgt aussehen: Jeder Mandatsbewerber - also auch Listenbewerber - muss in einem Wahlkreis kandidieren; jeder im Wahlkreis wahlberechtigte Bürger darf sich dort um eine Direkt- oder Listenkandidatur bewerben; statt der Nominierungsversammlungen der Parteien werden - unter staatlicher Aufsicht - von den Parteien organisierte Vorwahlen durchgeführt, in denen die Gewinner das Recht erwerben, bei der „eigentlichen“ Wahl für die jeweilige Partei anzutreten; an diesen Vorwahlen - und zwar bei allen Parteien - dürfen sich alle im Wahlkreis wahlberechtigten Bürger beteiligen. Zu allen Einzelheiten und zu den zu erwartenden Folgen siehe Werner J. Patzelt, Verbessern wir unsere repräsentative Demokratie! Ein Weg: Vorwahlen in Deutschland. Einige Thesen (Publikationsmanuskript, erhältlich vom Verfasser).

53 Laut dem ZDF-Politbarometer vom Oktober 2012 (Forschungsgruppe Wahlen, Politbarometer KW 43, S. 3) meinten 76 Prozent der Deutschen, Bundestagsabgeordnete sollten ihre Nebeneinkünfte vollständig offenlegen müssen; dagegen waren 20 Prozent. 
Sehr wohl müsse sich der „Wert eines Abgeordneten“ auch in dessen Bezahlung niederschlagen; doch seine Nebentätigkeiten habe er dann eben auf Euro und Cent genau offenzulegen. ${ }^{54}$ Und Donata Riedel, differenziert in ihrem Urteil über die Empfehlungen der Expertenkommission, schrieb ebenfalls in aller Klarheit: „Noch immer nicht ausreichend ist die Transparenz bei den Honoraren für Nebentätigkeiten: Wer eine ordentliche Abgeordnetendiät bekommt, sollte die Wähler schon vollständig über die Höhe der Nebeneinkünfte informieren müssen. "55 Ralf Dorschel spitzte das im Berliner Kurier vom 3. April 2013 so zu: „Nicht die Bezüge [der Abgeordneten] sind das Problem, sondern die Nebenjobs. Die Reden vor Finanzhaien, die Liebesdienste für Konzernlobbys. ... Wer als Volksvertreter 100.000 Euro im Jahr verdient, muss eben nicht mehr jeden Deal im Hinterzimmer annehmen."56 Alledem sekundierte Christian Humborg, Geschäftsführer von Transparency International Deutschland, mit der folgenden Bemerkung: „Je höher die Diäten, umso unverständlicher sind Nebeneinkünfte, die sich aus Tätigkeiten ergeben, die der Abgeordnete erst seit der Mandatsübernahme ausübt. " ${ }^{57}$ Aus diesem Zusammenhang heraus, zu Recht skandalisiert zwischen 2005 und 2007 angesichts der nachgewiesenen Verwicklung von je einem Bundestags- und Landtagsabgeordneten in die so genannte „VWKorruptionsaffäre" und einer Reihe weiterer Fälle ${ }^{58}$, ergibt sich auch einige Plausibilität für die - inzwischen erfüllte - Forderung, gemeinsam mit der Neuregelung der Abgeordnetenbezüge auch im Abgeordnetengesetz den Tatbestand der Abgeordnetenbestechung zu regeln. Alles in allem findet sich hier, wie bei der Altersversorgung und der Kostenpauschale, weiterhin eine Reformbaustelle unseres Abgeordnetenrechts.

\section{6. „Wo bleibt da die Gerechtigkeit!“}

Allem Anschein nach stehen nicht einfach nur antiparlamentarische Affekte ${ }^{59}$ hinter so vieler Kritik an den Vorschlägen der Expertenkommission. Oft wirkt da ein „Anti-ParteienAffekt" 60 in Verbindung mit generalisierter, also meist gerade nicht personalisierter, Verachtung für Berufspolitiker und Parlamentarier, die sich aus vielerlei Quellen speist. ${ }^{61}$ Doch

54 AFP-Meldung vom 3. April 2013.

55 Handelsblatt vom 3. April 2013.

56 Das muss man übrigens mit einem Gehalt (weit) unter 100.000 Euro auch nicht.

57 Zitiert nach Donata Riedel, Abgeordnetendiäten: Union bremst Lammert, in: Handelsblatt vom 3. April 2013, S. 11. In diesem Zusammenhang ist auf die langjährige, ungehörige und unlängst in Bayern zum Skandal und zum politischen Absturz unter anderem des CSU-Fraktionsvorsitzenden im Bayerischen Landtag führende Praxis hinzuweisen, nach der Abgeordnete Familienmitglieder ihm Rahmen ihrer Amtsausstattung beschäftigten und auf diese Weise ihr Familieneinkommen erhöhten; siehe hierzu auch den Beitrag von Heinrich Oberreuter in diesem Heft der ZParl.

58 Siehe hierzu Hans Leyendecker, Die große Gier. Korruption, Kartelle, Lustreisen. Warum unsere Wirtschaft eine neue Moral braucht, Berlin 2007, sowie - voller Polemik - Guido Grandt, Aktenzeichen Politiker. Die kriminellen Machenschaften deutscher Volksvertreter, Rottenburg 2009.

$59 \mathrm{Zu}$ deren Konstanten siehe Hartmut Wasser, Parlamentarismuskritik vom Kaiserreich zur Bundesrepublik. Analyse und Dokumentation, Stuttgart u.a. 1974.

60 Vgl. hierzu Gunter Hofmann, Die Kontroverse: Weizsäckers Parteienkritik in der Diskussion, Frankfurt am Main 1992.

61 Vgl. die Analyse von Jens Borchert, Die Professionalisierung der Politik. Zur Notwendigkeit eines Ärgernisses, Frankfurt am Main 2003. 
auch das ist nicht der letzte Kontext im Wahrnehmungs- und Deutungsgefüge. Letztlich geht es vielen Kritikern, wie beredt oder grobschlächtig auch immer formuliert, um Gerechtigkeit. In Alltagsgesprächen lauten die einschlägigen Fragen nicht selten so: Ist es wohl gerecht, wenn man nach wenigen Jahren im Parlament um soviel mehr bekommt als nach vielen Jahren in der Fabrik, oder wenn man schon in jungen Jahren ein Gehalt beziehen kann, das Richter und Kommunalpolitiker erst am Ende ihrer Laufbahn erhalten?

Gerade weil die Expertenkommission dem „Volksempfinden“ hier nicht nachgeben wollte, ja im vorangestellten „Leitbild“ auch noch klare Gründe für ihre ganz andere Position formulierte, lassen sich Bemerkungen wie die von Karl Doemens in seinem Kommentar über „Lammerts Diätenreform“ zunächst einmal nachvollziehen: „Eine solche Reform verletzt grob das Gerechtigkeitsempfinden der Gesellschaft. ${ }^{62}$ Offenbar konkurrieren hier sehr verschiedene Maßstäbe für Gerechtigkeit und gerechte Bezahlung. Den einen Maßstab leitet die Kommission aus den Anforderungen des Abgeordnetenamtes ab, zumal aus dessen Rolle für die Gesetzgebung - und der andere Maßstab gründet in jenen anscheinend weiterbestehenden Empfindungen, aus denen heraus die Grünen einst, nach ihrem erstmaligen Einzug in den Bundestag, ihre Parlamentarier durch „freiwillige Abgaben“ auf das Einkommensniveau von Facharbeitern bringen wollten.

Auf eine ernsthafte Zurkenntnisnahme, geschweige denn auf eine vergleichende Diskussion dieser konkurrierenden Gerechtigkeitsmaßstäbe ließ sich die am Bericht der Expertenkommission geäußerte Kritik aber nicht ein, sondern beließ es bei Bekundungen ihres Unmuts. Dabei hätte es sich sehr wohl angeboten, sich mit der Maßstabsbegründung der Kommission im „Leitbild“ und mit den daraus abgeleiteten Gehaltsempfehlungen sachlich auseinanderzusetzen. Dass dies unterblieb, zeigt einen wunden Punkt der Politischen Kultur an, ja mehr noch einen regelrechten „Wundbrand“. Solange nämlich das, was an den Abgeordnetenbezügen beibehalten oder neu eingeführt wird, einfach als ungerechtfertigter „Genuss von Privilegien“ verstanden wird, lässt sich die Verstetigung einer generalisierten Neiddebatte einfach nicht vermeiden. Deren Kollateralschäden sind wiederum Ansehensmängel der Abgeordneten bei der Bevölkerung. Reiner Holznagel, Präsident des Bundes der

62 Karl Doemens, „Lammerts Diätenreform“, a.a.O. (Fn. 17). Siehe hierzu auch den folgenden Blogeintrag auf „mitmischen.de“, dem Jugendportal des Bundestages: „Ich bin seit Juni 2013 Vollrentnerin $(65+2 \mathrm{Mt})$. Nach 40 Jahren versicherungspflichtiger Tätigkeit (kaufm- Angestellte - vorwiegend als Buchhalterin in Vollzeit) wurde mir eine Monatsrente von $€ 925,49$ pro Monat - BRUTTO zugewiesen. Ich möchte behaupten, dass ich mehr ,rotiert' und nicht minder Verantwortung getragen habe als einer, der nur mit dem Mund (debatieren) und herumsitzen, Hand hochheben und ähnlichen ,schweren' Arbeiten sein Kaviar-Brot verdienen muss. Meine Rente wurde nach langem herumdebatieren von solchen ,Schwerarbeitern' um € 2,31 (BRUTTO) mtl. erhöht! Ich habe mich noch nicht dafür bedankt! Entschuldigung! Diese ,Schwerstarbeiter 'verdienen monatlich neben Diäten + Pauschale und Vergünstigungen mehr als das 10-fache! Und jetzt haben sie unter sich weitere Erhöhung um über $€ 500,00 \mathrm{mtl} ! !$ beschlossen. Wieso fragen sie nicht uns, die Wähler, vorher ob wir das genehmigen? Ich habe keine Worte mehr und schon gar nicht ein Verständnis dafür!!" (Schreibfehler im Original). Ebenso klar ist auch folgender Eintrag vom 14. Februar auf Bild online Community: „Wenn diese selbstgefällige Selbstbedienung (Diäten / Steuerhinterziehung / Aufsichtsratsposten, schneller Wechsel in die Wirtschaft etc.) so weitergeht, und dann jemand den richtigen Nerv der Bevölkerung trifft, dann gibt es irgendwann RICHTIG Probleme, und zwar radikale, da die Bevölkerung sich immer mehr verkaspert und ausgeplündert vorkommt, wenn ein ,Normalo' bereis wegen geringer Steuerschuld bestraft wird. Die Verhältnismässigkeit stimmt einfach nicht mehr, was übrigens auch auf ALLE EU ABGEORDNETEN zutriff!!“ (Schreibfehler im Original). 
Steuerzahler, formulierte dies plakativ so: „Nur wenn endlich die Privilegien bei der Bezahlung unserer Politiker abgeschafft werden, kann die Politik auf das Vertrauen und die Akzeptanz der Bürger und Wähler hoffen."63

Gewiss ist der Zusammenhang zwischen Diäten und Vertrauen nicht so simpel, wie er in solcher Zuspitzung formuliert wird, denn während Holznagel allem Anschein nach behauptet, nur moderate Gehälter förderten Vertrauen, meint Annett Meiritz aus dem Bericht der Kommission ganz im Gegenteil herauszulesen: „Mehr Gehalt gleich mehr Respekt.“64 Doch grundsätzlich gibt es wohl Zusammenhänge, wenn sie auch sehr verwickelt sind. Insbesondere unter der Perspektive der „Leistungsgerechtigkeit“ verbindet sich der Blick auf den „Sold des Politikers" 65 mit einem Urteil darüber, was von den Volksvertretern zu halten wäre. Im „Originalton Volk“ klingen einschlägige Aussagen so:

„Diese Damen und Herren im ,Reich'stag haben unsere Rentenkassen geplündert, Schulden in gigantischer Höhe angehäuft, um anderen Ländern zu helfen, die Steuern in bisher nicht erreichte Höhe getrieben, Abgaben eingeführt, die den Bürgern den Hals abdrücken und der EU erlaubt, Deutschland mit einem Gängelband zu foltern, dass uns Bürgern die Zunge zum Hals raus hängt. Und nun sorgen sich die PolitikerInnen erst einmal um sich selbst und erhöhen ihre saftigen Pensionen. Prost! Zeit für eine Teaparty in Germany."66

Vor einem derartigen Wahrnehmungs- und Beurteilungshintergrund kann man dann auch gut nachvollziehen, was sich im Berliner Kurier vom 3. April 2013 unter dem Titel „,,6\% mehr Gehalt: Dreist oder gerecht?" als Zitat von der Webseite „Abgeordnetenwatch“ so lesen lässt: „In Zeiten von Nullrunden wäre eine weitere Erhöhung der Diäten nicht

63 Zitiert nach einer dapd-Meldung vom 4. April 2013. Wie sich dann die 2014 tatsächlich folgende Diätenerhöhung unter dem Gesichtspunkt der Gerechtigkeit für manchen anfühlte, geht aus dem folgenden Eintrag auf „Abgeordnetenwatch“ hervor, der unter dem Titel „Gier frisst Hirn“ am 26. Februar 2014 eingestellt wurde: „Was für eine Maßlosigkeit der Bundestagsabgeordneten während der Olympischen Spiele schnell ihre Diäten zu erhöhen. Fast 9.100 Euro im Monat!!! Das habe ich nicht einmal als Jahresrente! Eine Unverschämtheit und Diskriminierung von Menschen, die 4 Jahrzehnte arbeiteten dazu sich noch ehrenamtlich und gemeinnützig für Stadt, Kirche und Staat engagieren mit einer Armutsrente (Regelaltersrente $(65 \mathrm{~J})$ für langjährig Versicherte) von knapp über 600 Euro abzuspeisen, mit denen sie im Monat existieren sollen! Sozialschmarotzer, die keinen Handschlag für die Allgemeinheit tun, geht es besser wie uns. Sie erhalten Miete, Warmwasser, Heizung usw. bezahlt. Für alles müssen Rentner selbst aufkommen. Bei Anträgen auf Zuschuss wird nur die Grundmiete angesetzt, so dass der Rentner durchs Raster fällt und keinerlei Anspruch entsteht. Der Sozialschmarotzer erhält die volle Miete mit Heizkosten und Warmwasser. Außerdem gibt es in den neuen Bundesländern nach wie vor weniger Rentenpunkte! Keiner dieser Bundestagsabgeordneten fragt wie wir leben. auch Herr Hochbaum (Christlich Demokratische Union) stimmte für seine Erhöhung, obwohl er die Verhältnisse armer Rentner im Vogtland sehr gut kennt. Aber er schafft als Wessi lieber Erde aus dem Osten nach Berlin. Was für eine Lebensleistung. Wir alle, die die Werte schafften fühlen uns verraten, belogen und betrogen" (Schreibfehler im Original).

64 Annett Meiritz, Experten über Abgeordnete: Schlaflos, gestresst, unterbezahlt, in: Spiegel Online vom 4. April 2013, http://www.spiegel.de/politik/deutschland/diaeten-debatte-mehr-gehalt-fuermehr-respekt-a-892283.html (Abruf am 4. Juni 2014).

65 So der berühmt gewordene Titel von Theodor Eschenburg, Der Sold des Politikers, Stuttgart 1959.

66 Online-Kommentar auf der Webseite der Zeitung Die Welt zur Berichterstattung über den Abschlussbericht der Kommission vom 21. März 2013. Sozusagen ins Konstruktive wendet entsprechende Gerechtigkeitsforderungen ein Leserbriefschreiber im Focus vom 15. April 2013, S. 92: „Eine Erhöhung der Diäten darf erst nach Abbau der Schulden infrage kommen, dann haben die Volksvertreter endlich etwas in die ,richtige Richtung' geleistet." 
nachvollziehbar" 67 , ja schlechterdings ungerecht. Weil nun aber in unserem Staatswesen Gerechtigkeit nicht nur ein wichtiger Leitwert ist, sondern - wie irrtumsbeladen auch immer - Demokratie als Instrument für mehr Gerechtigkeit und Parlamentarismus als Konkretisierung von Demokratie verstanden wird, kann es gar nicht ausbleiben, dass jede vom Gerechtigkeitsargument befeuerte Kritik an Abgeordneten der Legitimität der parlamentarischen Demokratie Abbruch tut. Also müssen wir auf Möglichkeiten sinnen, zwar weiterhin das Abgeordnetenamt gemäß seiner öffentlichen Bedeutung zu dotieren, doch für eine Auswahl von solchen Parlamentariern zu sorgen, denen das Volk nicht das ihrer Leistung entsprechende Gehalt missgönnt.

\section{7. „Bekommen wir die, richtigen' Abgeordneten“?}

Diesen Gesichtspunkt vernachlässigen gerade jene wenigen Befürworter der Kommissionsvorschläge, die - wie der oben ausführlich zitierte - Peter Hahne ein vergleichsweise hohes Abgeordnetengehalt deshalb befürworten, weil es ein parlamentarisches Mandat auch für andere Berufsgruppen als „Beamte und Funktionäre“ attraktiv mache. Doch auf diese Weise werden die tatsächlichen Wirkungszusammenhänge des Systems der Abgeordnetenrekrutierung wohl verkannt ${ }^{68}$, denn nicht das Gehalt als solches scheint der in jungen Jahren zur Politik ziehende Magnet zu sein. Eine größere Rolle dürften Vorfreude auf Politik spielen, auch der Wunsch, die eigene politische Begabung zu erkunden, und obendrein die Chance, über politische Mandate auch sozial aufzusteigen. Hingegen ist vielen anderen, die - beruflich erfolgreich - um die Mitte ihres Lebens ins Parlament streben, der Weg dorthin, zumindest in den größeren Parteien, durch solche Konkurrenten versperrt, die seit Jugendtagen politisch aktiv waren. ${ }^{69}$ Insofern deutete Gregor Mayntz am zentralen Problem vorbei,

67 Hingegen als „nachvollziehbar“ gelten anscheinend die Nullrunden bei der Besoldung von Bundestagsabgeordneten zwischen 1977 bis 1983, 1992 bis 1995 und 2003 bis 2007 - vorausgesetzt, man weiß um sie.

$68 \mathrm{Zu}$ diesem siehe in einer den untersuchten Einzelfall weit übergreifenden Perspektive Pippa Norris / Joni Lovenduski, Political Recruitment. Gender, race and class in the British Parliament, Cambridge 1995.

69 Ein langjähriger Europaabgeordneter der CSU beschrieb 1989 - also 25 Jahre vor den heute sichtbaren sozialstrukturellen Ergebnissen des Karrierisierungsprozesses politischer Laufbahnen - diesen Zusammenhang so: „Ein Problem sehe ich darin, daß immer weniger Politiker oder (...) politischer Nachwuchs (...) aus Kreisen kommt, oder durch Persönlichkeiten kommt, die sich im bürgerlichen Leben bereits einen Namen gemacht haben oder durchgesetzt haben. (...) Eine der Gefährdungen in unserer parlamentarischen Demokratie besteht darin, daß es Karrierepolitiker von den politischen Jugendverbänden angefangen bis rauf gibt, die dann von zu früher Zeit [an] die Politik zu ihrem Lebensberuf gemacht haben" (zitiert nach Werner J. Patzelt, Abgeordnete und ihr Beruf. Interviews, Umfragen, Analysen, Berlin 1995, S. 313). Ein bayerischer Landtagsabgeordneter von der SPD sekundierte ihm damals wie folgt: „Seiteneinsteiger funktioniert nicht. Und zwar deswegen, weil Sie in der Organisation hinreichend [viele] nicht unqualifizierte Leute haben, die Parteiarbeit machen. Und es mag ja einer, der als Seiteneinsteiger in Frage käme, in der Qualifikation besser sein als der, der die Parteiarbeit mitmacht. Aber der Unterschied ist nicht so exorbitant, daß die Mitglieder in der Partei sagen würden, deswegen, weil der 30 Platzziffern im Examen vor dem andern war, würden wir den als Seiteneinsteiger akzeptieren. Das ist in allen Parteien so, glaube ich" (zitiert nach ebenda, S. 319). Viele der in jenem Band auf S. 265 - 269 und S. 311 - 321 umfänglich zitierten Abgeordneten diagnostizieren dieselbe Problemlage samt deren strukturellen, allem Anschein nach bis heute unveränderten Verursachungszusammenhän- 
als er in der Rheinischen Post vom 3. April 2013 meinte: „Der Tritt auf die Diäten-Bremse bedeutet dann auch: Immer mehr gerade der fähigsten Köpfe werden es sich nicht mehr leisten, für einige Jahre Politik zu machen.“

Gleiches gilt für Dorothea Siems, die in der „Welt" vom 3. April 2013 ebenfalls von der Rekrutierung und Selektion von Parlamentariern handelte. Auch sie zeigte nämlich keinen anderen - und zumal keinen konsensfähigeren - Lösungsweg auf als einen nach der Formel „ein hohes Gehalt, bitte!“. Denn sie meinte, es müsse ganz einfach ...

„die Bevölkerung interessiert daran sein, dass kluge und engagierte Menschen eine politische Karriere attraktiv finden. Und zwar nicht nur Berufseinsteiger, sondern auch gestandene Unternehmer, erfolgreiche Anwälte oder erfahrene Handwerksmeister. Dass heutzutage der öffentliche Dienst überrepräsentiert ist ${ }^{70}$ im Bundestag, liegt auch an den bescheidenen [sic!] Gehältern der Abgeordneten. Überdies gibt es etliche Politiker, die nie einen anderen Beruf aktiv ausgeübt haben und deren Erfahrungshorizont entsprechend eingeschränkt ist".

Siems blickt nämlich gar nicht auf die Eigentümlichkeiten unserer konkreten Abgeordnetenrekrutierung, wenn sie nach den - möglichst abzustellenden - Ursachen des von ihr beschriebenen Zustandes forscht. Dazu fällt ihr nämlich neben der „mäßigen Bezahlung“ vor allem, und zwar zu Recht, das „negative Image der Volksvertreter“ auf, das „den Job nicht attraktiver“ mache. An die Seite stellt sie diesem Hinderungsgrund dann auch noch den „geringen Gestaltungsspielraum“ von Politik. Letzterer aber lässt sich - gerade für die Abgeordneten (sub-) nationaler Parlamente unter den Umständen europäischer Integration ohnehin nicht erweitern, während sich das Gehalt von Politikern erst recht nicht steigern lässt, ohne unter den bestehenden Umständen das Ansehen der Abgeordneten noch problematischer zu machen. Der Ausweg muss also an anderer Stelle gesucht werden, als sie sowohl die Expertenkommission als auch die an ihren Vorschlägen geübte Kritik andeuten. ${ }^{71}$

\section{Politisch-kulturelle Tiefenschichten der Kritik}

Wie fügen sich nun die Einzelbefunde zur Kritik an den Vorschlägen der Expertenkommission zu einem größeren Ganzen, von dem her ihre Struktur verständlich wird - und das sie anders zu erklären erlaubte als durch den allzu schlichten Hinweis, dass viele Bürger in Be-

gen. Vor deren Hintergrund wird auch klar, warum die jetzige Abgeordnetenschaft der ostdeutschen Landesparlamente, ganz anders als während der ersten Wahlperioden, ihren westdeutschen Kollegen inzwischen sozialstrukturell so ähnlich geworden ist, obwohl rein rechtlich auch dort ein jeder in jeder Lebensphase versuchen kann, Parlamentarier zu werden. $\mathrm{Zu}$ den Motiven von ins Parlament führenden Laufbahnen siehe ebenda, S. 269 - 271; an neueren Studien über deutsche Parlamentskandidaten vgl. Marion Reiser, Wer entscheidet unter welchen Bedingungen über die Nominierung von Kandidaten? Die innerparteilichen Selektionsprozesse zur Aufstellung in den Wahlkreisen. Die Parteien nach der Bundestagswahl 2009, in: Oskar Niedermayer (Hrsg.), Die Parteien nach der Bundestagswahl 2009, Wiesbaden 2011, S. 237 - 259, sowie Suzanne S. Schüttemeyer / Roland Sturm, Der Kandidat - das (fast) unbekannte Wesen: Befunde und Überlegungen zur Aufstellung der Bewerber zum Deutschen Bundestag, in: ZParl, 36. Jg. (2005), H. 3, S. $539-553$.

70 Tatsächlich war das aber auch schon im (Früh-)Parlamentarismus des 19. Jahrhundert so; siehe etwa Heinrich Best, Parliamentary Representatives in Europe 1848-2000. Legislative recruitment and careers in eleven European countries, Oxford 2000.

71 Hierzu siehe den Vorschlag in Fußnote 52. 
zug auf Abgeordnete einfach ignorant oder böswillig wären? Am besten stellt man diese Einblicke in den Rahmen dessen, was inzwischen zu den Tiefenschichten bundesdeutscher Parlaments- und Abgeordnetenwahrnehmung bekannt ist. Das ist nicht wenig und beruht, neben Einzelbefunden verschiedener Meinungsforschungsinstitute ${ }^{72}$, der Studie von Dietrich Herzog über „Abgeordnete und Bürger“"73 sowie einigen neueren Studien ${ }^{74}$ nicht zuletzt auf zwei repräsentativen Umfragen des Verfassers aus den Jahren 1995 und 2004. Deren zentralen Befunde ${ }^{75}$ lassen sich so zusammenfassen:

- Die Deutschen kennen nicht nur ihre Parlamente abseits des Plenums als deren „Schauseite“ eher schlecht als recht, sondern haben auch noch einen ziemlich negativen Eindruck vom „institutionellen Charakter“ und von den Leistungen ihrer Parlamente, zumal dann, wenn es um Gemeinwohlorientierung versus Parteilichkeit, um Sachlichkeit und Gerechtigkeit sowie um so etwas wie „kostengünstige Effizienz“ geht.

- Das Bild der Deutschen vom Parlament und seinen Abgeordneten wird besonders stark von der aktuell wahrgenommenen allgemeinen politischen und wirtschaftlichen Lage geprägt, wobei letztere stets als viel schlechter denn die eigene wirtschaftliche Lage erscheint. ${ }^{76}$ Außerdem beeinflusst jenes Bild der - wohl nicht ganz entgegen der massenmedialen Berichterstattung - weit verbreitete Eindruck, die politische Klasse arbeite nicht so recht gemäß den Wünschen des Volkes, ja kümmere sich nicht einmal sonderlich um sie. Deutlich weniger wird das Bild des Parlaments und der Parlamentarier aber davon beeinflusst, was der Parlamentarismus im Lauf der Jahrzehnte insgesamt für Deutschland geleistet hat, zumal, angesichts der Trümmerlandschaft des Kriegsendes, mit der Schaffung und Weiterentwicklung der Voraussetzungen für eine vorzüglich funktionierende Wirtschaft und Demokratie. Und noch weniger wird das Bild der Deutschen vom Parlament und seinen Abgeordneten geprägt durch die Wahrnehmung oder Wertschätzung jener Werte und Prinzipien, auf deren Verwirklichung gerade die parlamentarische Demokratie ausgeht - vom pluralistischen Meinungsstreit bis hin zur Schaffung von Gemeinwohl durch Kompromissbildung.

72 Für die ersten Jahrzehnte unserer parlamentarischen Demokratie finden sie sich zusammengetragen und interpretiert in: Suzanne S. Schüttemeyer, Bundestag und Bürger im Spiegel der Demoskopie. Eine Sekundäranalyse zur Parlamentarismusperzeption in der Bundesrepublik Deutschland, Opladen 1986.

73 Dietrich Herzog / Hilke Rebenstorf / Camilla Werner / Bernhard Weßels, Abgeordnete und Bürger. Ergebnisse einer Befragung der Mitglieder des 11. Deutschen Bundestages und der Bevölkerung, Wiesbaden 1990.

74 Siehe etwa Mirjam Dageförde, Weit entfernt vom „idealen Abgeordneten“? Zu Normen und Praxis parlamentarischer Repräsentation aus Sicht der Bürger, in: ZParl, 44. Jg. (2013), H. 3, S. 580 - 592; Elisa Deiss-Helbig, Ich bin einer von Euch - Zur Bedeutung sozialer und politischer Kongruenz von Abgeordneten und Bürgern für das Gelingen von Repräsentation, in: ZParl, 44. Jg. (2013), H. 3, S. 566 - 580; Oscar W. Gabriel / Lisa Schöllhammer, Warum die Deutschen ihren Abgeordneten nicht mehr vertrauen als dem Bundestag, in: ZParl, 40. Jg. (2009), H. 2, S. $414-$ 430.

75 Beide Umfragen wurden von der DFG finanziert. Die zentralen Publikationen sind: Werner J. Patzelt, Ein latenter Verfassungskonflikt?, a.a.O. (Fn. 20) und ders., Warum verachten die Deutschen ihr Parlament, a.a.O. (Fn. 20).

76 Letzteres zeigen seit Jahrzehnten die regelmäßig im „Politbarometer“ der Forschungsgruppe Wahlen veröffentlichten Befunde. 
- Die populäre Wahrnehmung von Parlamenten und Abgeordneten hängt stark von der Themensetzung und Themenrahmung der Massenmedien ab, die ihrerseits aber - und zwar ganz gemäß journalistischer Nachrichtenwertlogik und Kritikfunktion - besonders stark das Misslingende, Ungenügende und Skandalöse betonen. Wenn aber gilt „Nur schlechte Nachrichten (über Parlamentarier) sind gute Nachrichten“, dann muss es auch nicht wundern, wenn das populäre Abgeordnetenbild deutlich negativ gefärbt ist. $^{77}$

- Die Deutschen schätzen zwar die ihnen persönlich bekannten Parlamentarier durchaus gut ein, zumal Abgeordnete aus der eigenen Region sowie fernsehbekannte Spitzenpolitiker der eigenen Couleur. Sie tun dies aber eher gefühlsmäßig und jedenfalls ohne sonderliches Wissen um deren konkrete Arbeit. Dabei vermeiden sie kognitive Dissonanzen oder den Eindruck unbegründeten Meinens in der Regel dadurch, dass sie immerhin die Illusion von Wissen pflegen. Das gelingt, indem sie es mit der Nutzung jener Denk- und Redefiguren bewenden lassen, die im Alltagsgespräch widerspruchslos, ja oft sogar beifällig „durchgehen“, weil sie aktuell von den Massenmedien in Geltung gehalten oder im Bildungsprozess als „Selbstverständlichkeiten“ vermittelt werden. Bei Abgeordneten betrifft derlei vor allem deren „Abgehobenheit“, deren Agieren als „Marionetten von Lobbyisten“, die „Bevormundung durch ihre Parteien“ sowie, ein ums andere Mal, ihre als erwiesen geltende „Geldgier“.

- Nicht nur die Praxis des Parlamentarismus, sondern auch die für ihn wichtigen Spielregeln werden von vielen Deutschen verkannt. Davon betroffen sind gerade Kernelemente des demokratischen Parlamentarismus: die für pluralistische Demokratie erforderliche offene Konfliktaustragung („Die streiten doch nur!“) samt anschließender Kompromissbildung („faule Kompromisse“, „die stecken doch alle unter einer Decke!“); die Arbeitsteiligkeit parlamentarischer Willensbildung und Entscheidungsfindung („Im Parlament [gemeint ist so gut wie immer: im Plenum] reden die doch gar nicht ernsthaft zur Sache, wenn sie überhaupt hingehen!"); sowie die Entstehung und Verwirklichung von Fraktionsdisziplin („Das sind doch alles nur brave Fraktionssoldaten!“). Deshalb überziehen sehr viele Bürger sogar das ordnungsgemäße Funktionieren des Bundestages und ein ganz korrektes Verhalten der Abgeordneten mit ganz aufrichtig als zutreffend empfundener Kritik.

- Die Deutschen mögen insbesondere die Parteien nicht, weshalb sie unter den politischen Akteuren beziehungsweise Institutionen alle jene ziemlich verachten, die - wie der Bundestag und die Bundesregierung - sehr stark mit Parteien oder mit Parteipolitik zu tun haben. Eben das zeigen regelmäßig die „Vertrauensranglisten“ politischer Institutionen: Oben stehen immer die überparteilichen Institutionen wie Bundespräsident und Bundesverfassungsgericht - ganz unten hingegen die Parteien sowie, da unverkennbar von Parteipolitikern geprägt, Bundestag und Bundesregierung.

77 In so einem Verständnisrahmen wirken dann aber auch ganz sachliche Informationen ziemlich anders, als sie das in jenem Verständnisrahmen tun, den etwa die Expertenkommission mit ihrem „Leitbild“ anbot. Peter Hahne - oben ausführlich zitiert - erlebte also im Grund nichts anderes als einen Konflikt der Nutzer von zwei sehr verschiedenen Verständnisrahmen des Parlamentarismus. In diesem Konflikt zu unterliegen ist dann besonders schmerzlich, wenn man gute Gründe für die Annahme hat, gerade der eigene Verständnisrahmen sei angemessen. 
Bei einer auf solchen politisch-kulturellen Tiefenschichten fußenden Parlaments- und Abgeordnetenwahrnehmung muss es aber nicht wundern, wenn die Deutschen wenig Vertrauen zu ihren Abgeordneten haben, ihnen ihr Einkommen missgönnen und gern ignorieren, was die Parlamentarismusforschung und das auf ihr fußende „Leitbild“ der Expertenkommission ihnen über Abgeordnete und ihren Beruf nahebringen wollen. Einmal mehr legt das die Einsicht nahe, dass nicht das politisches System und auch nicht die Politiker der Schwachpunkt unserer Demokratie sind, sondern populäre Missverständnisse und Vorurteile, die das parlamentarische Regierungssystem samt den Abgeordneten recht anders erscheinen lassen, als es tatsächlich ist. Eben das kam - einmal mehr - recht unverfälscht in der populären Kritik an den Empfehlungen der „Diätenkommission“ zum Ausdruck. Also sollte man diese Kritik sehr wohl beherzigen - nur ziemlich anders, als das viele der Kritiker im Sinn hatten.

\title{
Funktionszulagen für Parlamentarische Geschäftsführer. Zum Urteil des Schleswig-Holsteinischen Landesverfassungsgerichts vom 30. September $2013^{1}$
}

\author{
Siegfried Jutzi
}

\section{Hintergrund}

In seinem so genannten ersten Diätenurteil (1975) leitete das Bundesverfassungsgericht (BVerfG) für den Sachbereich der Wahlen aus dem Gleichheitssatz und „der historischen Entwicklung zum Demokratisch-Egalitären hin, die im Grundgesetz für das Bundestagswahlrecht in Art. 38 Abs. 1 Satz 1 und für das Wahlrecht in den Ländern, Kreisen und Gemeinden in Art. 28 Abs. 1 Satz 2 ihren verfassungsrechtlich verbindlichen Ausdruck gefunden hat" ${ }^{\text {“2 }}$ ab, „daß jedem Abgeordneten eine gleich hoch bemessene Entschädigung zusteht, unabhängig davon, ob die Inanspruchnahme durch die parlamentarische Tätigkeit größer oder geringer ist, ob der individuelle finanzielle Aufwand oder das berufliche Einkommen verschieden hoch ist "3. Eine Ausnahme von dem solchermaßen formalisierten Gleichheitssatz erkannte das Gericht lediglich für Parlamentspräsidenten und ihre Stellvertreter an, da sie „an der Spitze eines obersten Verfassungsorgans stehen “4. Das Urteil erfuhr von Anfang an, unter anderem wegen seines ,egalitären Rigorismus “" ${ }^{\text {, }}$, deutliche Kritik. ${ }^{6}$

1 Schleswig-Holsteinisches LVerfG, Urteil vom 30. September 2013, Az. LVerfG 13/12.

2 BVerfGE 40, S. 296, S. 317.

3 Ebenda, S. 318.

4 Ebenda.

5 Klaus Stern, Das Staatsrecht der Bundesrepublik Deutschland, Bd. I, München 1984, \$ 24 II 1, S. 1057.

6 Vgl. nur Hans Hugo Klein, in: Theodor Maunz / Günter Dürig (Hrsg.), Grundgesetz, Kommentar, Art. 48 (Stand: 12/2007), Rn. 168; Stefanie Schmahl, Funktionszulagen - ein Verstoß gegen Mandatsfreiheit und Gleichheit der Abgeordneten?, in: AöR 2005, S. 114 - 149, S. 131 ff.; an- 